\title{
Semistability of the tangent sheaf of singular varieties
}

\author{
Henri Guenancia
}

\begin{abstract}
The main goal of this paper is to prove the polystability of the logarithmic tangent sheaf $\mathscr{T}_{X}(-\log D)$ of a $\log$ canonical pair $(X, D)$ whose canonical bundle $K_{X}+D$ is ample, generalizing in a significant way a theorem of Enoki. We apply this result and the techniques involved in its proof to get a Bogomolov-type inequality for Chern numbers for log canonical pairs, a version of the semistability theorem for stable varieties (the higher-dimensional analogue of Deligne-Mumford's stable curves) and finally the generic semipositivity of the sheaf of logarithmic differentials of a log canonical pair with pseudo-effective log canonical class, in the spirit of Miyaoka's theorem.
\end{abstract}

\section{Introduction}

\subsection{Semistability}

This paper deals with the notion of slope semistability for coherent sheaves on singular varieties using a differential-geometric approach. This notion was first introduced by Mumford [Mum63] in his attempt to construct bounded families of vector bundles over a curve. Recall that a vector bundle $E$ over a complex projective curve $C$ is said to be semistable (respectively, stable) if for every proper subbundle $F$ of $E$, we have

$$
\frac{\operatorname{deg}(F)}{\operatorname{rk} F} \leqslant \frac{\operatorname{deg}(E)}{\operatorname{rk} E} \quad\left(\text { respectively, } \frac{\operatorname{deg}(F)}{\operatorname{rk} F}<\frac{\operatorname{deg}(E)}{\operatorname{rk} E}\right)
$$

where the degree of $F$ is $\int_{C} c_{1}(F)$; the quantity $\operatorname{deg}(F) / \operatorname{rk} F$ is called the slope of $F$ and denoted $\mu(F)$. When one tries to generalize this definition to higher-dimensional varieties, one first needs an ample line bundle $H$ (called a polarization) to define a degree and thus a slope: $\operatorname{deg}_{H}(F)=c_{1}(F) \cdot H^{n-1}$ and $\mu_{H}(F)=\operatorname{deg}_{H}(F) / \mathrm{rk} F$. And more importantly, in order to keep the good moduli properties shared by stable vector bundles over curves, one needs to ask that not only all subbundles of $E$ have slope smaller than that of $E$, but also all its proper coherent subsheaves; cf. Definition 3.1.

Received 5 April 2015, accepted in final form 7 January 2016.

2010 Mathematics Subject Classification 32C20, 32Q20, 32W20, $53 \mathrm{C} 07$.

Keywords: slope stability, stable varieties, log canonical pairs, generic semipositivity, normal Kähler spaces, complex Monge-Ampère equations, singular Kähler-Einstein metrics, Bogomolov inequality.

This journal is (C) Foundation Compositio Mathematica 2016. This article is distributed with Open Access under the terms of the Creative Commons Attribution Non-Commercial License, which permits non-commercial reuse, distribution, and reproduction in any medium, provided that the original work is properly cited. For commercial re-use, please contact the Foundation Compositio Mathematica.

The author is partially supported by NSF Grant DMS-1510214. 


\subsection{The Kobayashi-Hitchin correspondence}

One of the major developments regarding stable vector bundles is the celebrated KobayashiHitchin correspondence, relating the stability of a vector bundle $E$ (an algebro-geometric property) to the existence of a special hermitian metric on $E$ (a differential-geometric property).

KobAYASHI-Hitchin CORRESPONDENCE. Let $E$ be a holomorphic vector bundle on a compact Kähler manifold $(X, \omega)$. Then $E$ is polystable with respect to $\omega$ if and only if $E$ admits a HermiteEinstein metric with respect to $\omega$.

Recall that a Hermite-Einstein metric $h$ on $E$ is a Hermite-Einstein metric with respect to a Kähler form $\omega$ if its Chern curvature tensor $\Theta_{h}(E) \in \mathscr{C}^{\infty}\left(X, \Omega^{1,1} \otimes \operatorname{End}(E)\right)$ satisfies $\operatorname{tr}_{\omega}\left(\Theta_{h}(E)\right)=\mu \operatorname{Id}_{E}$ for some constant $\mu \in \mathbb{R}$. Here, polystability means that $E$ is semistable and is the sum of stable subsheaves (that automatically have the same slope).

This correspondence is due to Kobayashi-Lübke [KobS82, Lüb83] in the direction "HermiteEinstein $\Rightarrow$ stable", to Donaldson for Riemann surfaces [Don83], algebraic surfaces [Don85] and manifolds [Don87] and to Uhlenbeck-Yau [UY86, UY89] for Kähler manifolds.

The case where $E=T_{X}$ is the tangent bundle of the manifold is of particular importance because due to the symmetry of the Riemann curvature tensor, if $\omega$ is a Kähler form and $h$ is the induced hermitian metric on $T_{X}$, then $\left(T_{X}, h\right)$ is Hermite-Einstein with respect to $\omega$ if and only if $\omega$ is a Kähler-Einstein metric, that is, $\operatorname{Ric} \omega=\mu \omega$ for some constant $\mu \in \mathbb{R}$. For many reasons, the Kähler-Einstein equation is in general easier to study or solve than the Hermite-Einstein equation, and currently we dispose of many existence results and tools in Kähler-Einstein theory. For example, when $K_{X}$ is ample (respectively, numerically trivial), the celebrated theorems of Aubin-Yau and Yau [Aub78, Yau78] provide a unique negatively curved Kähler-Einstein metric (respectively, Ricci-flat metric) on $X$ living in $c_{1}\left(K_{X}\right)$ (respectively, in any Kähler cohomology class). Bringing these theorem together with the Kobayashi-Hitchin correspondence, we obtain the following.

Corollary. Let $X$ be a compact Kähler manifold. Then

(i) if $K_{X}$ is ample, then $T_{X}$ is polystable with respect to $K_{X}$;

(ii) if $K_{X}$ is numerically trivial, then $T_{X}$ is polystable with respect to any Kähler class.

\subsection{Singular varieties and stability}

In the present paper, we will strive to extend the above result in a singular setting, pushing further the very elegant approach of Enoki [Eno88], who essentially proved the semistability of the tangent sheaf of $X$ in both cases above assuming merely that $X$ has canonical singularities. His strategy is to use approximate Kähler-Einstein metrics on a resolution $X^{\prime}$. By considering them as approximate Hermite-Einstein metrics on $T_{X^{\prime}}$, he obtains an "approximate slope inequality". Thanks to various estimates (due to Yau) about Monge-Ampère equations, he manages to control the error term in the inequality and to prove that it converges to zero at the end of the approximation process.

In a related though somewhat different framework, Tsuji [Tsu88] proved the semistability of the tangent bundle of smooth minimal projective manifolds (that is, with nef canonical bundle) using a perturbation argument involving conic metrics along some suitable divisor whose angle tends to $2 \pi$. He also states (without proof though) that the result holds as well for a log smooth pair $(X, D)$ such that $K_{X}+D$ is nef. 


\section{H. Guenancia}

We will generalize Enoki's theorem from three different perspectives: we will merely require that $X$ has $\log$ canonical singularities, we will allow a boundary divisor and work in the setting of log pairs, and finally we will show polystability in addition to semistability.

Theorem A. Let $X$ be a compact Kähler space and $D$ a reduced Weil divisor such that $(X, D)$ has $\log$ canonical singularities.

(i) If $K_{X}+D$ is ample, then the logarithmic tangent sheaf $\mathscr{T}_{X}(-\log D)$ is polystable with respect to $K_{X}+D$.

(ii) If $K_{X}+D \equiv 0$, then $\mathscr{T}_{X}(-\log D)$ is semistable with respect to any Kähler class. Moreover, if $D=0$ and $X$ has Kawamata log terminal (klt) singularities, then $\mathscr{T}_{X}$ is actually polystable with respect to any Kähler class.

Note that the ampleness of $K_{X}+D$ can be replaced by nefness without any change in the proof (for the semistability, not the polystability though); however, it is not that common to consider stability with respect to non-movable classes, which is why we left it out of the statement.

Compared to the proof in the case of canonical singularities, many serious new difficulties pop up in this general setting, mainly because the presence of the boundary divisor $D$ requires us to work with cuspidal metrics instead of smooth metrics. Also, passing from canonical to log canonical singularities depends upon a finer analysis of the regularity for solutions of degenerate Monge-Ampère equations, which we develop in Section 2. We have outlined the new issues we had to overcome in Section 3.2.

We should mention that Theorem A(ii) is closely linked with the main result of [GKP11], which further investigates the tangent sheaf of varieties with canonical singularities and vanishing first Chern class. Also, some of the techniques used in the proof appear in the very interesting paper [CP14], where one of the clever ideas to treat boundary problems is to use conic metrics (with angles going to zero); we will apply these ideas in the last section.

\subsection{Stable varieties}

Theorem A(i) can be used to deduce properties about the so-called stable varieties. In short, stable varieties are the higher-dimensional analogue of the stable curves introduced by Deligne and Mumford, in the sense that they allow us to compactify the moduli space of canonically polarized manifolds (say with fixed Hilbert polynomial). Their definition involves three requirements: projectivity, positivity of the canonical bundle and specific singularities; cf. Section 4.1 and the references therein for a more detailed account. For the time being, we just need to know that if $X$ is a stable variety, then $K_{X}$ can be defined as an ample $\mathbb{Q}$-line bundle, and the normalization map $\nu: X^{\nu} \rightarrow X$ satisfies $\nu^{*} K_{X}=K_{X^{\nu}}+D$, where $D$ is a reduced Weil divisor such that the pair $\left(X^{\nu}, D\right)$ has $\log$ canonical singularities.

Therefore, Theorem A guarantees that $\mathscr{T}_{X^{\nu}}(-\log D)$ is semistable with respect to $K_{X^{\nu}}+D$, and one can easily infer from this that $\operatorname{Aut}(X)$ is a finite group; cf. Corollary 3.6. One can also ask whether one can say anything about the semistability of the tangent sheaf of $X$ itself. Inspired by the case of stable curves, we introduce the sheaf $\nu_{*} \mathscr{T}_{X^{\nu}}(-\log D)$ that should be the "tangent sheaf" we want to look at in this setting, where the philosophy is to describe the objects upstairs. For non-normal varieties, the notions of slope and stability exist, but are way more delicate to deal with. We recall them in Section 4 and manage to prove the following result, which is a non-trivial consequence of Theorem A. 
Theorem B. Let $X$ be a stable variety, $\nu: X^{\nu} \rightarrow X$ its normalization map and $D \subset X^{\nu}$ the conductor of $\nu$. Then the sheaf $\nu_{*} \mathscr{T}_{X^{\nu}}(-\log D)$ is semistable with respect to $K_{X}$.

The proof of this theorem requires us to understand the relationship between the slope of a sheaf and that of its pull-back under the normalization map, which happens to be trickier than one could imagine at first sight; cf. Remark 4.8.

\subsection{Generic semipositivity}

The Miyaoka generic semipositivity theorem [Miy87] asserts that if $X$ is a normal projective variety, then $\Omega_{X}^{1}$ is generically semipositive unless $X$ is uniruled. Recall that a reflexive sheaf $\mathscr{E}$ on a normal projective variety $X$ is generically semipositive if for any ample polarizations $H_{1}, \ldots, H_{n-1}$ and any coherent quotient $\mathscr{F}$ of $\mathscr{E}$, the degree of $\mathscr{F}$ with respect to $\left(H_{1}, \ldots, H_{n-1}\right)$ is non-negative, that is, $\left(c_{1}(\mathscr{F}) \cdot H_{1} \cdots \cdot H_{n-1}\right) \geqslant 0$. This celebrated result had many applications and was recently generalized to the case of $\log$ smooth pairs $(X, D)$ such that $K_{X}+D$ is pseudoeffective by Campana-Păun [CP13, CP14]. Capitalizing on their method and the techniques of this paper, we are able to extend this to the following singular case.

Theorem C. Let $X$ be an $n$-dimensional compact Kähler space, $D$ a reduced Weil divisor such that $(X, D)$ has $\log$ canonical singularities and $\omega$ a Kähler form. If $K_{X}+D$ is pseudo-effective (respectively, $-\left(K_{X}+D\right)$ is pseudo-effective), then $\Omega_{X}^{1}(\log D)$ (respectively, $\mathscr{T}_{X}(-\log D)$ ) is generically $\omega$-semipositive.

In particular, if $K_{X}+D \equiv 0$, the slope of the logarithmic tangent sheaf vanishes, so the generic semipositivity of $\Omega_{X}^{1}(\log D)$ is equivalent to its semistability with respect to any Kähler class. This is how we will prove Theorem A(ii).

\subsection{A Bogomolov-type inequality}

In the last section of this paper, we investigate applications of the Kähler-Einstein theory for singular spaces to Chern numbers inequalities. In the smooth case, this is a very fruitful domain (cf. Section 6), but for singular varieties, the lack of understanding of the Kähler-Einstein metrics near the singularities makes it very hard to tackle such global questions. However, the point of this article is to show that even limited control over the singularities of the metric lets us prove semistability properties. Therefore, one can apply the Bogomolov-Gieseker inequality (generalized by Miyaoka) to our semistable logarithmic tangent sheaf in the context we are working in.

More precisely, let $(X, D)$ be a projective log canonical pair of dimension $n$, where $D$ is a reduced divisor. Let $\pi: Y \rightarrow X$ be a log resolution of the pair $(X, D)$; in particular, $\pi^{*} D+\operatorname{Exc}(\pi)$ is a divisor with simple normal crossings (snc) support. We denote by $\Delta$ the sum of the strict transform of $D$ and the exceptional divisor with zero log discrepancy; to put it in a different way, $K_{Y}+\Delta-\pi^{*}\left(K_{X}+D\right)$ is an exceptional divisor with coefficients strictly greater than -1 .

Theorem 1.1. Let $(X, D)$ be a projective log canonical pair as above. We have the following Bogomolov-type inequalities:

(i) If $K_{X}+D$ is ample, then

$$
c_{1}\left(T_{Y}(-\log \Delta)\right)^{2} \cdot\left(\pi^{*}\left(K_{X}+D\right)\right)^{n-2} \leqslant \frac{2 n}{n-1} c_{2}\left(T_{Y}(-\log \Delta)\right) \cdot\left(\pi^{*}\left(K_{X}+D\right)\right)^{n-2} .
$$




\section{H. Guenancia}

(ii) If $K_{X}+D \equiv 0$, then for any ample line bundle $H$ on $X$, we have

$$
c_{1}\left(T_{Y}(-\log \Delta)\right)^{2} \cdot\left(\pi^{*} H\right)^{n-2} \leqslant \frac{2 n}{n-1} c_{2}\left(T_{Y}(-\log \Delta)\right) \cdot\left(\pi^{*} H\right)^{n-2} .
$$

Of course, if the non-snc locus $(X, D)_{\text {sing }}$ has codimension greater than 2 , then we can define $c_{1}$ and $c_{2}$ for the logarithmic tangent sheaf $\mathscr{T}_{X}(-\log D)$ as the push-forwards of the usual cycles on $(X, D)_{\text {reg }}$ by the open immersion. If $K_{X}+D$ is ample, the projection formula yields

$$
c_{1}\left(\mathscr{T}_{X}(-\log D)\right)^{2} \cdot\left(K_{X}+D\right)^{n-2} \leqslant \frac{2 n}{n-1} c_{2}\left(\mathscr{T}_{X}(-\log D)\right) \cdot\left(K_{X}+D\right)^{n-2},
$$

and if $K_{X}+D \equiv 0$, it yields

$$
c_{1}\left(\mathscr{T}_{X}(-\log D)\right)^{2} \cdot H^{n-2} \leqslant \frac{2 n}{n-1} c_{2}\left(\mathscr{T}_{X}(-\log D)\right) \cdot H^{n-2} .
$$

\subsection{Organization of the paper}

In Section 2 we obtain a Laplacian estimate for solutions of Monge-Ampère equations with degenerate right-hand side, which will turn out to be crucial for the proof of the semistability. In Section 3 we first recall the definitions of stability, log canonical singularities and logarithmic tangent sheaf; then we state the first part of Theorem A, explain the difficulties and prove it. In Section 4 we give the definition of stable varieties as well as semistability in a context of non-normal varieties, and then prove Theorem B. In Section 5, extending the techniques from Campana-Păun's papers [CP13, CP14] to the singular case, we prove generic semipositivity for the sheaf of logarithmic differentials assuming that the logarithmic canonical bundle is pseudoeffective. Then we apply this result to prove Theorem A(ii). In Section 6 we prove a Bogomolovtype inequality for Chern numbers on log canonical pairs.

\section{A Laplacian estimate}

This section is devoted to the technical Proposition 2.1, which can be seen as a generalization of the Laplacian estimate obtained using the Chern-Lu formula. It will be used in the next section to control the error term in the semistability inequality.

So let us first recall the Chern-Lu formula [Che68, Lu68], which is going to be a essential tool to get the Laplacian estimate. Let $\left(X, \omega_{X}\right)$ and $\left(Y, \omega_{Y}\right)$ be two Kähler manifolds and $f: X \rightarrow Y$ a holomorphic map satisfying $\partial f \neq 0$. Then

$$
\Delta_{\omega_{X}} \log |\partial f|^{2} \geqslant \frac{\operatorname{Ric} \omega_{X} \otimes \omega_{Y}(\partial f, \overline{\partial f})}{|\partial f|^{2}}-\frac{\omega_{X} \otimes R^{Y}(\partial f, \overline{\partial f}, \partial f, \overline{\partial f})}{|\partial f|^{2}},
$$

where $\partial f$ is viewed as a section of $T_{X}^{*} \otimes f^{*} T_{Y}$.

Using this formula, when $f$ is the identity map (but the Kähler forms differ), one can derive so-called Laplacian estimates for the Kähler-Einstein equation provided that the reference metric has holomorphic bisectional curvature bounded from above (cf. [JMR16, Section 7]). The following proposition, inspired by [Pău08] (see also [BBEGZ11, Theorem 10.1]), enables us to derive (weaker) Laplacian estimates in some cases where the Ricci curvature is not bounded from below.

Proposition 2.1. Let $X$ be a compact Kähler manifold of dimension $n$, and let $\omega$ and $\omega^{\prime}$ be two cohomologous Kähler metrics on $X$. We assume $\omega^{\prime}=\omega+d d^{c} \varphi$, that there exists a smooth function $\Psi$ and that we have a constant $C>0$ satisfying 
(i) $\sup _{X}|\varphi| \leqslant C$,

(ii) $\sup _{X} \Psi \leqslant C$ and $d d^{c} \Psi \geqslant-C \omega$,

(iii) $\Theta_{\omega}\left(T_{X}\right) \leqslant C \omega \otimes \operatorname{Id}_{T_{X}}$,

(iv) $\operatorname{Ric} \omega^{\prime} \geqslant-C \omega^{\prime}-C \omega-d d^{c} \Psi$.

Then there exists some constant $M>0$ depending only on $n$ and $C$ such that

$$
\omega^{\prime} \geqslant M^{-1} e^{\Psi} \omega
$$

In the above proposition, we used the operator $d^{c}:=\frac{1}{2 i \pi}(\partial-\bar{\partial})$, and we denoted by $\Theta_{\omega}\left(T_{X}\right)$ the Chern curvature of the hermitian vector bundle $\left(T_{X}, \omega\right)$. The inequality in part (iii) is to be understood in the sense of Griffiths semipositivity.

Proof. The main difficulty is that we do not have control over the lower bound on Ric $\omega^{\prime}$. The trick, inspired by [Pău08], is to add $\psi$ in the Laplacian appearing in the Chern-Lu formula. Let us now get into the details. We apply the Chern-Lu formula to $f=\mathrm{id}:\left(X, \omega^{\prime}\right) \rightarrow(X, \omega)$. Then $|\partial f|^{2}=\operatorname{tr}_{\omega^{\prime}} \omega$. We denote by $g$ and $h$ the hermitian metrics induced by $\omega^{\prime}$ and $\omega$, respectively. The second term of the Chern-Lu formula is easily dealt with:

$$
\begin{aligned}
-\omega^{\prime} \otimes R^{h}(\partial f, \overline{\partial f}, \partial f, \overline{\partial f}) & =-g^{i \bar{j}} g^{k \bar{l}} R_{i \bar{j} k \bar{l}}^{h} \\
& \geqslant-C g^{i \bar{j}} g^{k \bar{l}}\left(h_{i \bar{j}} h_{k \bar{l}}+h_{i \bar{l}} h_{k \bar{j}}\right) \\
& \geqslant-2 C\left(\operatorname{tr}_{\omega^{\prime}} \omega\right)^{2} .
\end{aligned}
$$

To analyze Ric $\omega^{\prime} \otimes \omega(\partial f, \bar{\partial} f)$, it is convenient to introduce a suitable system of coordinates. More precisely, at a given point $p \in X$, one can choose geodesic coordinates such that $\left(h_{k \bar{l}}(0)\right)$ is diagonal, $g_{i j}(0)=\delta_{i j}$, and the first-order derivatives of $g_{i j}$ vanish at $p$ (cf., for example, [Bło12, (1.2)-(1.4)]). So, at $p$, we have

$$
\begin{aligned}
\operatorname{Ric} \omega^{\prime} \otimes \omega(\partial f, \overline{\partial f}) & =\sum_{i, j, k, l} g^{i \bar{k}} g^{j \bar{l}} R_{i \bar{j}}^{g} h_{k \bar{l}} \\
& \geqslant-\sum_{i, j, k, l} g^{i \bar{k}} g^{j \bar{l}}\left(C g_{i \bar{j}}+\left(C h_{i \bar{j}}+\Psi_{i \bar{j}}\right)\right) h_{k \bar{l}} \\
& =-\sum_{i}\left(C g^{i \bar{i}}+g^{i \bar{i}} g^{i \bar{i}}\left(C+\Psi_{i \bar{i}}\right)\right) \\
& \geqslant-C\left(\sum_{i} g^{i \bar{i}}\right)-\left(\sum_{i} g^{i \bar{i}}\right) \cdot\left(\sum_{j} g^{j \bar{j}}\left(C+\Psi_{j \bar{j}}\right)\right) \\
& =-C \operatorname{tr}_{\omega^{\prime}} \omega-\left(\operatorname{tr}_{\omega^{\prime}} \omega\right) \cdot \operatorname{tr}_{\omega^{\prime}}\left(C \omega+d d^{c} \Psi\right) .
\end{aligned}
$$

At that point, the Chern-Lu formula gives us

$$
\Delta_{\omega^{\prime}} \log \operatorname{tr}_{\omega^{\prime}} \omega \geqslant-3 C \operatorname{tr}_{\omega^{\prime}} \omega-C-\operatorname{tr}_{\omega^{\prime}} d d^{c} \Psi,
$$

and therefore

$$
\Delta_{\omega^{\prime}}\left(\log \operatorname{tr}_{\omega^{\prime}} \omega+\Psi\right) \geqslant-3 C \operatorname{tr}_{\omega^{\prime}} \omega-C .
$$

Setting $A=3 C+1$, we get, as usual,

$$
\Delta_{\omega^{\prime}}\left(\log \operatorname{tr}_{\omega^{\prime}} \omega+\Psi-A \varphi\right) \geqslant \operatorname{tr}_{\omega^{\prime}} \omega-n A-C .
$$




\section{H. Guenancia}

The end is classic: we choose a point $p$ where $\log \operatorname{tr}_{\omega^{\prime}} \omega+\Psi-A \varphi$ attains its maximum; then

$$
\begin{aligned}
\log \operatorname{tr}_{\omega^{\prime}} \omega & \leqslant\left(\log \operatorname{tr}_{\omega^{\prime}} \omega+\Psi-A \varphi\right)(p)-\Psi+A \varphi \\
& \leqslant(\log (n A+C)+\sup \Psi+2 A \sup |\varphi|)-\Psi,
\end{aligned}
$$

which gives the expected result since we have a uniform bound on $\|\varphi\|_{\infty}$, by assumption.

Remark 2.2. Note that it is not clear a priori how to deduce the previous estimate using [Pău08] by exchanging the roles of $\omega$ and $\omega^{\prime}$, because we would no longer have control over the bisectional curvature of $\omega^{\prime}$.

Combining the previous result and Păun's estimate, we obtain the following estimate.

Corollary 2.3. Let $X$ be a compact Kähler manifold of dimension $n$, and let $\omega$ and $\omega^{\prime}$ be two cohomologous Kähler metrics on $X$. We assume $\omega^{\prime}=\omega+d d^{c} \varphi$ with $\omega^{\prime n}=e^{\psi^{+}-\psi^{-}} \omega^{n}$ for some smooth functions $\psi^{ \pm}$, and that we have a constant $C>0$ and some $p>1$ satisfying

(i) $\sup _{X} \psi^{+} \leqslant C$ and $\left\|e^{-\psi^{-}}\right\|_{L^{p}\left(\omega^{n}\right)} \leqslant C$,

(ii) $d d^{c} \psi^{ \pm} \geqslant-C \omega$.

Then there exists some constant $M>0$ depending only on $n, p, C$ and $\omega$ such that

$$
M^{-1} e^{\psi^{+}} \omega \leqslant \omega^{\prime} \leqslant M e^{-\psi^{-}} \omega .
$$

\section{Polystability of the logarithmic tangent sheaf}

\subsection{Generalities}

In this section, we briefly recall the definitions of slope, semistability, various types of singularities appearing in the minimal model program, as well as the (logarithmic) tangent sheaf before presenting previously known results. We refer to [Har80], [KobS87, Chapter V] or [HL10] for more details.

Notion of stability. In the following, $X$ will be a complex projective normal variety of dimension $n$ (or a normal Kähler space), and $\mathscr{F}$ will always denote a coherent sheaf. We set $r=$ rk $\mathscr{F}$, and we let $\operatorname{det} \mathscr{F}:=\left(\Lambda^{r} \mathscr{F}\right)^{* *}$ be the determinant of $\mathscr{F}$. It is a rank 1 reflexive sheaf on $X$, but if $X$ is not smooth, it is in general not a line bundle; that is, it is not locally free. We will denote by $c_{1}(\mathscr{F})$ the equivalence class of any Weil divisor attached to det $\mathscr{F}$. We can now define the slope.

Definition 3.1. Let $H$ be an ample line bundle on $X$. We define the slope of $\mathscr{F}$ with respect to $H$ to be the rational number

$$
\mu_{H}(\mathscr{F}):=\frac{c_{1}(\mathscr{F}) \cdot H^{n-1}}{\operatorname{rk} \mathscr{F}} .
$$

The same definition can be transposed without any change if $H$ is merely the coholomogy class of a Kähler form in a normal Kähler space. Let us now give the definition of slope stability, which goes back to Mumford [Mum63] and Takemoto [Tak72].

Definition 3.2. Let $\mathscr{E}$ be a torsion-free coherent sheaf on $X$ and $H$ an ample line bundle. We say that $\mathscr{E}$ is semistable (respectively, stable) with respect to $H$ if for every coherent subsheaf $\mathscr{F} \subset \mathscr{E}$ (respectively, every non-zero and proper coherent subsheaf $\mathscr{F}$ ), we have

$$
\mu_{H}(\mathscr{F}) \leqslant \mu_{H}(\mathscr{E}) \quad\left(\text { respectively }, \mu_{H}(\mathscr{F})<\mu_{H}(\mathscr{E})\right) .
$$




\section{SEMISTABILITY OF THE TANGENT SHEAF OF SINGULAR VARIETIES}

We say that $\mathscr{E}$ is polystable (with respect to $H$ ) if $\mathscr{E}$ is the direct sum of stable subsheaves with the same slope.

Canonical and log canonical singularities. Let us now very briefly give a definition of the class of singularities we are going to deal with (see [KM98] for a more detailed account).

Definition 3.3. Let $X$ be a complex normal variety, $D$ an effective Weil $\mathbb{Q}$-divisor $D$ such that $K_{X}+D$ is $\mathbb{Q}$-Cartier and $\pi: X^{\prime} \rightarrow X$ a log resolution of $(X, D)$. We define the coefficients $a_{i}$ by the formula

$$
K_{X^{\prime}}=\pi^{*}\left(K_{X}+D\right)+\sum a_{i} E_{i},
$$

where $E_{i}$ is either an exceptional divisor or the strict transform of a component of $D$.

- If $D=0$, we say that $X$ has canonical (respectively, log terminal) singularities if for all $i$, one has $a_{i} \geqslant 0$ (respectively, $a_{i}>-1$ ).

- Else, we say that the pair $(X, D)$ has klt (respectively, log canonical) singularities if for all $i$, one has $a_{i}>-1$ (respectively, $a_{i} \geqslant-1$ ).

All these singularities are normal, so that the notions of slope and semistability make sense for these varieties.

The logarithmic tangent sheaf. Let us first recall that on an arbitrary complex variety $X$, one can define the sheaf $\Omega_{X}^{1}$ of Kähler differentials (cf. [Har77, II.8]) and define the tangent sheaf $\mathscr{T}_{X}$ of $X$ as the dual $\left(\Omega_{X}^{1}\right)^{*}$ of the sheaf of Kähler differentials. If $X$ is smooth, then this sheaf corresponds to the sheaf associated with the tangent bundle $T_{X}$. If $X$ is merely normal, then $\mathscr{T}_{X}$ is a reflexive sheaf, so that by the observation above, it can equivalently be defined as the push-forward of $\mathscr{T}_{X_{\text {reg }}}$ via the open immersion $X_{\text {reg }} \hookrightarrow X$.

Let us now consider log pairs. Let $X$ be a normal projective variety and $D$ a reduced Weil divisor on $X$. If the pair $(X, D)$ is $\log$ smooth, that is, $X$ is smooth and $D$ is reduced with simple normal crossings, we have a well-defined logarithmic tangent bundle $T_{X}(-\log D)$ which is the dual of the bundle of logarithmic differentials $\Omega_{X}^{1}(\log D)$. It simply consists of vector fields that vanish along $D$; that is, if $D$ is locally given by $\left(z_{1} \cdots z_{k}=0\right)$, then the sheaf at stake is the locally free $\mathcal{O}_{X}$-module generated by

$$
z_{1} \frac{\partial}{\partial z_{1}}, \ldots, z_{k} \frac{\partial}{\partial z_{k}}, \frac{\partial}{\partial z_{k+1}}, \ldots, \frac{\partial}{\partial z_{n}} .
$$

To define its analogue in a more general setting, we proceed as follows. We denote by $(X, D)_{\text {reg }}$ the snc locus of the pair $(X, D)$, that is, the locus of points $x \in X$ where $(X, D)$ is $\log$ smooth in a neighborhood of $x$. As $X$ is normal, it is smooth in codimension 1, and $D$ is generically smooth; therefore $(X, D)_{\text {reg }}$ is a Zariski-open set whose complement has codimension at least 2 . Let us denote by $j:(X, D)_{\text {reg }} \hookrightarrow X$ the open immersion.

Definition 3.4. Let $(X, D)$ be a $\log$ pair as above, and denote by $U:=(X, D)_{\text {reg }}$ its snc locus. The logarithmic tangent sheaf of $(X, D)$, denoted by $\mathscr{T}_{X}(-\log D)$, is defined as $j_{*} T_{U}\left(-\log D_{\mid U}\right)$. Its dual is denoted $\Omega_{X}^{1}(-\log D)$ and is called the sheaf of (reflexive) logarithmic differentials.

The sheaf $\mathscr{T}_{X}(-\log D)$ (as well as its dual) is automatically coherent, and hence reflexive by, for example, [Har80, Proposition 1.6]. One can equivalently define the logarithm tangent sheaf as the sheafification of the module of derivations that preserve the ideal sheaf corresponding to $D$. 


\section{H. Guenancia}

When $(X, D)$ is log smooth, the logarithmic tangent sheaf is locally free, and we will denote the corresponding vector bundle by $T_{X}(-\log D)$.

In the case where $X$ is merely a compact normal Kähler space, one needs to be more careful, as $j_{*} T_{U}\left(-\log D_{\mid U}\right)$ is not automatically coherent, unlike in the projective case. However, one can always consider the sheafification of the module of derivations that preserve the ideal sheaf corresponding to $D$. This is an analytic coherent sheaf on $X$ that extends $T_{U}\left(-\log D_{\mid U}\right)$, and therefore, by the main result of [Ser66], the sheaf $j_{*} T_{U}\left(-\log D_{\mid U}\right)$ is itself a (reflexive) analytic coherent sheaf. We denote it by $\mathscr{T}_{X}(-\log D)$, and call it the logarithmic tangent sheaf of $(X, D)$. Its dual, $\Omega_{X}^{1}(-\log D)$, is called the sheaf of (reflexive) logarithmic differentials.

\subsection{Statement of the result}

Our main result extends Enoki's theorem (say, when $K_{X}$ is ample) to the case of a log canonical pair $(X, D)$ and also proves the polystability.

Theorem 3.5. Let $X$ be a normal projective variety and $D$ a reduced Weil divisor such that $(X, D)$ is $\log$ canonical and $K_{X}+D$ is an ample $\mathbb{Q}$-line bundle. Then $\mathscr{T}_{X}(-\log D)$ is polystable with respect to $K_{X}+D$.

Compared to Enoki's setting, in this generalized setting many new difficulties arise that require much fine analysis and some recent work:

- getting precise estimates for the Monge-Ampère equations with a very degenerate righthand side (this is the content of Lemma 3.7, which is a combination of Proposition 2.1 and [GW16, Theorem A])

- working with cuspidal metrics instead of smooth ones (which requires the delicate Proposition 3.8)

- analyzing the limiting behavior of the approximate Kähler-Einstein metrics (we will use [BG14] and [GW16, Theorem B] in a crucial way) to get polystability

However, these complications come from the relatively large degree of generality that we chose to work with, and we could have obtained weaker generalizations of Enoki's theorem at a lower cost; cf. Remark 3.10.

\subsection{An application to automorphism groups}

A first application of Theorem 3.5 concerns the finiteness of some automorphism groups. Indeed, it is well known that semistable sheaves with negative slopes do not admit any non-trivial sections, and relying on this fact and the above theorem, one can prove the following.

Corollary 3.6. The automorphism group $\operatorname{Aut}(X)$ of a stable variety $X$ is finite.

For the definition of a stable variety, see Definition 4.1. The finiteness of the automorphism group of such varieties was already well known; cf. [Miy83, BHPS13] or [BG14, Theorem 6.3] for a differential-geometric proof in the normal case.

Proof of Corollary 3.6. Let $\nu: Y \rightarrow X$ be the normalization of $X$. By the universal property of the normalization, any automorphism of $X$ can be lifted to an automorphism of $Y$, giving an injection $i: \operatorname{Aut}(X) \rightarrow \operatorname{Aut}(Y)$; observe that any element in $i(\operatorname{Aut}(X))$ preserves the conductor $\Delta$ of the normalization. Moreover, the subgroup of $\operatorname{Aut}(Y)$ fixing every connected component of $Y$ has finite index in $\operatorname{Aut}(Y)$, so we can assume that $Y$ is connected. 
So we are reduced to showing that for a normal variety $Y$ and a reduced divisor $\Delta$ such that the pair $(Y, \Delta)$ has $\log$ canonical singularities and $K_{Y}+\Delta$ is ample, the automorphism group $\operatorname{Aut}(Y, \Delta)$ of the pair $(Y, \Delta)$ is finite; this group is defined as the (closed) subgroup of $\operatorname{Aut}(Y)$ fixing $\Delta$. But any such automorphism will preserve $K_{Y}+\Delta$, and therefore our group is a subgroup of the automorphism of a polarized variety, so it is a linear group (as it embeds into $\operatorname{PGL}(N, \mathbb{C})$ for some large integer $N)$. Therefore $\operatorname{Aut}(Y, \Delta)$ is finite if and only if its tangent space is trivial, but its tangent space is precisely $H^{0}\left(Y, \mathscr{T}_{Y}(-\log \Delta)\right)$, the space of holomorphic vector fields tangent to $\Delta$. Indeed, given a 1-parameter family of such automorphisms $\left(\phi_{t}\right)$, each of them preserves $(Y, \Delta)_{\text {reg, }}$, the snc locus of the pair, so taking the derivative at $t=0$ yields a vector field on $(Y, \Delta)_{\text {reg }}$ tangent to $\Delta$. As $\left.\mathscr{T}_{Y}(-\log \Delta)\right)$ is reflexive and the complement of $(Y, \Delta)_{\text {reg }}$ has codimension at least 2 , we get an element in $H^{0}\left(Y, \mathscr{T}_{Y}(-\log \Delta)\right)$. The correspondence works just as well in the other direction by restricting the vector field to $(Y, \Delta)_{\text {reg }}$ and taking its flow, which extends to an automorphism of the pair $(Y, \Delta)$ by [BBEGZ11, Lemma 5.2].

Now, any non-zero element $\xi \in H^{0}\left(Y, \mathscr{T}_{Y}(-\log \Delta)\right)$ induces an injective sheaf morphism $f: \mathcal{O}_{Y} \rightarrow \mathscr{T}_{Y}(-\log \Delta)$ (the kernel is a torsion sheaf, but $\mathcal{O}_{Y}$ is torsion free), hence $f\left(\mathcal{O}_{Y}\right)$ is a subsheaf of $\mathscr{T}_{Y}(-\log \Delta)$ with zero slope. This contradicts the semistability of $\mathscr{T}_{Y}(-\log \Delta)$, whose slope $-\left(K_{Y}+\Delta\right)^{n} / n$ is strictly negative.

\subsection{Proof of Theorem A}

Proof of Theorem 3.5. We proceed in four steps.

Step 1. Reduction to the log smooth case. Let $(Y, \Delta)$ be a log canonical pair with $\Delta$ a reduced Weil divisor such that $K_{Y}+\Delta$ is ample, and let $\mathscr{G}$ be a coherent subsheaf of $\mathscr{T}_{Y}(-\log \Delta)$ of rank $r>0$. We choose a log resolution $\pi: X \rightarrow Y$ of the pair that is an isomorphism over its snc locus; we denote by $\Delta^{\prime}$ the strict transform of $\Delta$. There exists a $\pi$-exceptional divisor $E=\sum a_{i} E_{i}$ such that $K_{X}+\Delta^{\prime}=\pi^{*}\left(K_{Y}+\Delta\right)+E$, and we have $a_{i} \geqslant-1$ for all $i$ by the log canonical assumption. Let us set $F=\sum_{a_{i}=-1} E_{i}$, the "purely $\log$ canonical part" and $D:=\Delta^{\prime}+F$; for reasons that will appear later, we want to find a coherent subsheaf $\mathscr{F}$ of $\mathscr{T}_{X}(-\log D)$ such that $\pi_{*} c_{1}(\mathscr{F})=c_{1}(\mathscr{G})$. We consider the snc locus $(Y, \Delta)_{\text {reg }}$ of the pair, which is an open subset of $Y$ whose complement has codimension at least 2 , and we set $U:=\pi^{-1}\left((Y, \Delta)_{\text {reg }}\right)$ and let $j: U \rightarrow X$ denote the open immersion. Then the sheaf $\mathscr{F}:=\left(j_{*}\left(\pi^{*} \mathscr{G}\right)_{\mid U}\right) \cap \mathscr{T}_{X}(-\log D)$ is a coherent subsheaf of $\mathscr{T}_{X}(-\log D)$ that satisfies $\pi_{*} c_{1}(\mathscr{F})=c_{1}(\mathscr{G})$ on $(Y, \Delta)_{\text {reg }}$, hence on the whole $Y$.

Finally, as $c_{1}\left(\mathscr{T}_{Y}(-\log \Delta)\right)$ is represented by $-\left(K_{Y}+\Delta\right)$, the projection formula yields the following equivalences:

$$
\begin{aligned}
& \frac{c_{1}(\mathscr{G}) \cdot\left(K_{Y}+\Delta\right)^{n-1}}{r} \leqslant \frac{c_{1}\left(\mathscr{T}_{Y}(-\log \Delta)\right) \cdot\left(K_{Y}+\Delta\right)^{n-1}}{n} \\
\Longleftrightarrow \quad \frac{c_{1}(\mathscr{F}) \cdot \pi^{*}\left(K_{Y}+\Delta\right)^{n-1}}{r} & \leqslant \frac{-\pi^{*}\left(K_{Y}+\Delta\right)^{n}}{n} \\
\Longleftrightarrow \quad & \frac{c_{1}(\mathscr{F}) \cdot \pi^{*}\left(K_{Y}+\Delta\right)^{n-1}}{r} \leqslant \frac{-\left(K_{X}+\Delta^{\prime}-E\right) \cdot \pi^{*}\left(K_{Y}+\Delta\right)^{n-1}}{n} \\
& \quad \frac{c_{1}(\mathscr{F}) \cdot \pi^{*}\left(K_{Y}+\Delta\right)^{n-1}}{r} \leqslant \frac{c_{1}\left(\mathscr{T}_{X}(-\log D)\right) \cdot \pi^{*}\left(K_{Y}+\Delta\right)^{n-1}}{n},
\end{aligned}
$$

as $E$ is $\pi$-exceptional.

Therefore we are reduced to showing the semistability of $\mathscr{T}_{X}(-\log D)$ with respect to 


\section{H. Guenancia}

$\pi^{*}\left(K_{Y}+\Delta\right)$. Actually, we will prove a bit more and show that for every generically injective sheaf morphism $\mathscr{F} \rightarrow \mathscr{T}_{X}(-\log D)$, we have the expected slope inequality.

Step 2. Construction of appropriate cusp metrics. Let us first introduce some notation. We choose an ample line bundle $A$ on $X$, a Kähler form $\omega_{A}$ whose cohomology class is $c_{1}(A)$, and a Kähler form $\omega_{0}$ on $Y$ representing $c_{1}\left(K_{Y}+\Delta\right)$. We set $X_{0}:=X \backslash D$, and we fix two parameters $\varepsilon, t>0$ for our regularization process. Finally, we consider for each $i$ such that $a_{i}>-1$ a regularizing family $\left(\theta_{i, \varepsilon}\right)_{\varepsilon>0}$ of $(1,1)$-forms in $c_{1}\left(a_{i} E_{i}\right)$ such that $\theta_{i, \varepsilon}$ converges to the singular current $a_{i}\left[E_{i}\right]$ when $\varepsilon$ goes to zero. An explicit formula for $\theta_{i, \varepsilon}$ can be given in terms of hermitian metrics $h_{i}$ on $\mathcal{O}_{X}\left(E_{i}\right)$ as well as sections $s_{i}$ cutting out the divisors $E_{i}$ :

$$
\theta_{i, \varepsilon}=a_{i}\left(\frac{\varepsilon^{2}\left|D_{i}^{\prime} s_{i}\right|^{2}}{\left(\left|s_{i}\right|^{2}+\varepsilon^{2}\right)^{2}}+\frac{\varepsilon^{2} \theta_{E_{i}}}{\left|s_{i}\right|^{2}+\varepsilon^{2}}\right)
$$

where $D_{i}^{\prime}$ is the $(1,0)$-part of the Chern connection of $\left(\mathcal{O}_{X}\left(E_{i}\right), h_{i}\right)$ and $\theta_{E_{i}}$ is its curvature. In the following, we will work with the metric $\omega$ satisfying

$$
\operatorname{Ric} \omega=-\omega+t \omega_{A}+[D]-\sum_{a_{i}>-1} \theta_{i, \varepsilon} .
$$

This metric $\omega$ belongs to $c_{1}\left(\pi^{*}\left(K_{Y}+\Delta\right)+t A\right)$ and depends on $\varepsilon$ and $t$, but we choose not to mention its dependence to keep the notation lighter. It is smooth on $X_{0}$ and has cusp singularities along $D$; that is, around points where $D$ is given by $\left(z_{1} \cdots z_{r}=0\right)$, the metric $\omega$ is uniformly equivalent to the model cusp metric (also called Poincaré metric)

$$
\sum_{k=1}^{r} \frac{i d z_{k} \wedge d \bar{z}_{k}}{|z|^{2} \log ^{2}|z|^{2}}+\sum_{k>r} i d z_{k} \wedge d \bar{z}_{k}
$$

The existence (and uniqueness) of $\omega$ is guaranteed by results of Kobayashi [KobR84] and TianYau [TY87], who obtained it as the solution of the Monge-Ampère equation

$$
\left(\pi^{*} \omega_{0}+t \omega_{A}+d d^{c} \varphi\right)^{n}=\frac{e^{\varphi+f} \prod_{i}\left(\left|s_{i}\right|^{2}+\varepsilon^{2}\right)^{a_{i}}}{\prod_{j}\left|t_{j}\right|^{2}} \omega_{A}^{n},
$$

where $f$ is some smooth function determined by $\omega_{A}$ as well as the hermitian metrics chosen on $E$ and $D,\left(s_{i}=0\right)$ cuts out $E_{i}$ for $i$ such that $a_{i}>-1$, and $\left(t_{j}=0\right)$ cuts out the $j$ th component of $D$. Moreover, one can rewrite this equation in a perhaps more standard form using the potential $\varphi_{P}=-\sum_{j} \log \log ^{2}\left|t_{j}\right|^{2}$ of the cusp metric along $D$ : setting $\omega_{P}:=\pi^{*} \omega_{0}+t \omega_{A}+d d^{c} \varphi_{P}$ and $\psi:=\varphi-\varphi_{P}$, the equation becomes

$$
\left(\omega_{P}+d d^{c} \psi\right)^{n}=\prod_{i}\left(\left|s_{i}\right|^{2}+\varepsilon^{2}\right)^{a_{i}} e^{\psi+F} \omega_{P}^{n},
$$

where $F$ is a smooth function when read in the quasi-coordinates; cf. [KobR84] or [Gue14, Lemma 4.3]. The metric $\omega=\omega_{P}+d d^{c} \psi$ (that depends strongly on $\varepsilon$ and $t$ ) satisfies the following property, which will be a cornerstone of the proof.

Lemma 3.7. For every fixed $t>0$ and any section $s=s_{i}$ or $s=t_{j}$, the integral

$$
\int_{X_{0}} \frac{\varepsilon^{2}}{|s|^{2}+\varepsilon^{2}} \omega_{A} \wedge \omega^{n-1}
$$

converges to zero when $\varepsilon$ goes to zero. 


\section{SEMistability of THE TANGENT SHEAF OF SINGUlaR VARIETIES}

Proof. Assume for the moment that we can prove that there is a constant $C$ depending only on $t>0$ such that

$$
\omega \geqslant C^{-1} \prod_{a_{i}>0}\left(\left|s_{i}\right|^{2}+\varepsilon^{2}\right)^{a_{i}} \omega_{P}
$$

In view of equation (3.3), satisfied by $\omega$, and the above assumption, we have

$$
\operatorname{tr}_{\omega}\left(\omega_{A}\right) \omega^{n} \leqslant C \frac{\prod_{a_{i}>-1}\left(\left|s_{i}\right|^{2}+\varepsilon^{2}\right)^{a_{i}} e^{\psi} \omega_{P}^{n}}{\prod_{a_{i}>0}\left(\left|s_{i}\right|^{2}+\varepsilon^{2}\right)^{a_{i}}}
$$

for some $C>0$ independent of $\varepsilon$ (depending on $\sup |F|$ and a constant $M>0$ such that $\left.\omega_{A} \leqslant M \omega_{P}\right)$. We need here another non-trivial input, as we want to get rid of $\psi$. This is possible by invoking [GW16, Theorem A] (or, more precisely, the outcome of Sections 3.3 and 3.4, where the Monge-Ampère equation studied is exactly (3.3) with $t>0$ fixed) that provides a bound $\sup |\psi| \leqslant C$ independent of $\varepsilon$ (but depending on $t$ ).

Combining these observations, we get

$$
\frac{\varepsilon^{2}}{|s|^{2}+\varepsilon^{2}} \omega_{A} \wedge \omega^{n-1} \leqslant \frac{C \varepsilon^{2}}{|s|^{2}+\varepsilon^{2}} \cdot \prod_{-1<a_{i}<0}\left(\left|s_{i}\right|^{2}+\varepsilon^{2}\right)^{a_{i}} \omega_{P}^{n} .
$$

Checking the convergence to zero of our integral is now local on $\operatorname{Supp}(D+E)$. As this divisor has simple normal crossing support, we can use Fubini's theorem to reduce our problem to a 1-dimensional one. We will be done once we have proved that both integrals

$$
\int_{\mathbb{D}} \frac{\varepsilon^{2} i d z \wedge d \bar{z}}{\left(|z|^{2}+\varepsilon^{2}\right)^{1+\delta}} \quad \text { and } \quad \int_{\mathbb{D}} \frac{\varepsilon^{2} i d z \wedge d \bar{z}}{\left(|z|^{2}+\varepsilon^{2}\right) \cdot|z|^{2} \log ^{2}|z|^{2}}
$$

converge to zero for any $\delta \in(0,1)$, where $\mathbb{D}$ is a small disc centered at $0 \in \mathbb{C}$. For the first integral, we can perform the change of variable $w=z / \varepsilon$, giving the integral

$$
\varepsilon^{2(1-\delta)} \int_{|w|^{2} \leqslant 1 / \varepsilon} \frac{|d w|^{2}}{\left(1+|w|^{2}\right)^{1+\delta}},
$$

which goes to zero as $1>\delta>0$. As for the second one, we know that $\varepsilon^{2}\left(|z|^{2}+\varepsilon^{2}\right)^{-1} \leqslant 1$ and that $\left(|z|^{2} \log ^{2}|z|^{2}\right)^{-1} \in L^{1}(\mathbb{D})$; we then just have to apply the dominated convergence theorem to conclude.

The only thing left to prove now is (3.4), and we would like to use Proposition 2.1 with $\omega^{\prime}=\omega$ and $\omega=\omega_{P}$. So we check the four conditions. For condition (i), we write $\omega=\omega_{P}+d d^{c} \psi$, where the $L^{\infty}$ estimate on $\psi$ comes from [GW16, Section 2.3-2.4]. For condition (iii), it is well known that the reference metric $\omega_{P}$ has bounded geometry so, in particular, it has a bounded curvature tensor. As for conditions (ii) and (iv), we set $\Psi:=\sum_{a_{i}>0} a_{i} \log \left(\left|s_{i}\right|^{2}+\varepsilon^{2}\right)$; it obviously satisfies condition (ii) and (outside $D$ ) we moreover have

$$
\begin{aligned}
\operatorname{Ric} \omega & \geqslant-\omega+t \omega_{A}-d d^{c} \sum_{a_{i}>0} a_{i} \log \left(\left|s_{i}\right|^{2}+\varepsilon^{2}\right)-\left(\sum_{-1<a_{i}<0} \frac{a_{i} \varepsilon^{2} \theta_{E_{i}}}{\left|s_{i}\right|^{2}+\varepsilon^{2}}+\sum_{a_{i}>0} a_{i} \theta_{E_{i}}\right) \\
& \geqslant-\omega-C \omega_{P}-d d^{c} \sum_{a_{i}>0} a_{i} \log \left(\left|s_{i}\right|^{2}+\varepsilon^{2}\right) \\
& =-\omega-C \omega_{P}-d d^{c} \Psi
\end{aligned}
$$

for some sufficiently big constant $C$. Therefore, inequality (3.4) would follow from Proposition 2.1 if we could establish it for metrics $\omega, \omega^{\prime}$ without the compactness assumption. It turns out that in 


\section{H. Guenancia}

our situation, the reference metric $\omega_{P}$ has bounded curvature tensor, and the unknown metric $\omega$ is complete with (qualitatively) bounded Ricci curvature (even bounded holomorphic bisectional curvature), so that we can apply the generalized maximum principle of Yau and mimic the proof of Proposition 2.1 without any serious change. This concludes the proof of (3.4), and hence of the lemma.

Step 3. Computing the slopes using the singular metrics. We start with a generically injective morphism $\mathscr{F} \rightarrow \mathscr{T}_{X}(-\log D)$. If $r=\operatorname{rk}(\mathscr{F})$, then this morphism induces $j: \operatorname{det} \mathscr{F} \rightarrow$ $\Lambda^{r} \mathscr{T}_{X}(-\log D)$, where, by definition, $\operatorname{det} \mathscr{F}:=\left(\Lambda^{r} \mathscr{F}\right)^{* *}$. The strategy consists of endowing $\mathscr{T}_{X}(-\log D)$ and $\mathscr{F}$ with the hermitian metrics induced by $\omega$ and computing the slopes of these sheaves using the corresponding representatives of their first Chern classes. Two different types of difficulty appear, though: First, as $\omega$ is cuspidal along $D$, it induces a singular hermitian metric on $\mathscr{T}_{X}(-\log D)$, and it is not clear that one can use it to compute any slope (whether for $\mathscr{T}_{X}(-\log D)$ or $\left.\mathscr{F}\right)$. Second, as $\omega$ is not Kähler-Einstein but only approximately KählerEinstein, it is not clear that the error term in the slope inequality will vanish when the various regularizing parameters (essentially $\varepsilon$ and $t$ ) will converge to zero.

We start by addressing the first difficulty. In order to do so, we introduce the singularity set $W=W(\mathscr{F})$, which is defined as the smallest analytic subset of $X$ outside which the sheaf morphism $\mathscr{F} \rightarrow \mathscr{T}_{X}(-\log D)$ is an injection of vector bundles. We denote by $F$ the vector bundle on $X \backslash W$ such that $\mathscr{F}=\mathcal{O}_{X}(F)$ there Without loss of generality, one can assume that $\mathscr{F}$ is saturated in $\mathscr{T}_{X}(-\log D)$ (it is enough to consider such subsheaves to test the semistability), so that $W$ has codimension at least 2 in $X$.

We denote by $h$ the smooth hermitian metric induced by $\omega$ on $T_{X}(-\log D)$ over $X \backslash D$. It also induces a smooth hermitian metric on $F$ over $X \backslash(W \cup D)$, that we still denote by $h$. We claim that $h$ can be used to compute the slope of $\mathscr{F}$ :

Proposition 3.8. We have

$$
\int_{X \backslash(W \cup D)} c_{1}(F, h) \wedge \omega^{n-1}=c_{1}(\mathscr{F}) \cdot\left(\pi^{*}\left(K_{Y}+\Delta\right)+t A\right)^{n-1} .
$$

Proof. This is far from being obvious, especially because of the singularities of $\omega$ along $D$. On $X \backslash(W \cup D)$, the metric $h$ induces a hermitian metric $h^{\wedge r}$ on $\Lambda^{r} F$ that we aim to compare with a smooth hermitian metric $h_{0}$ on $\operatorname{det} \mathscr{F}$ - notice that $\Lambda^{r} F$ and $\operatorname{det} \mathscr{F}$ coincide on $X \backslash(W \cup D)$. We are now going to analyze the behavior of $h^{\wedge r} / h_{0}$ on $W \cup D$. More precisely, if $\xi$ denotes a local trivialization of the line bundle det $\mathscr{F}$, then the quantity $h^{\wedge r}(\xi) / h_{0}(\xi)$ is independent of the choice of $\xi$, so it induces a smooth positive function $H$ on $X \backslash(W \cup D)$, and obviously $d d^{c} \log H=$ $c_{1}\left(\operatorname{det} \mathscr{F}, h_{0}\right)-c_{1}(F, h)$, so everything boils down to showing $\int_{X \backslash(W \cup D)} d d^{c} \log H \wedge \omega^{n-1}=0$. For the sake of clarity, we will distinguish three cases, even if they could be treated in a unified manner.

Case 1. Near a point $x \in W \backslash D$, the morphism $j: \operatorname{det} \mathscr{F} \rightarrow \Lambda^{r} T_{X}(-\log D)$ is degenerate. Choose trivializations of all bundles at stake. Then there are holomorphic function $\left(f_{1}, \ldots, f_{p}\right)$, where $p=\left(\begin{array}{l}n \\ r\end{array}\right)$, such that $j=\left(f_{1}, \ldots, f_{p}\right)$. Then $H=|j|_{h}^{2}$ is the squared norm (for some hermitian product on $\mathbb{C}^{p}$ ) of a vector of holomorphic functions, and we claim that $\log H$, possibly after a modification, is the sum of a smooth function and a pluriharmonic function outside $(j=0)$ which is nothing else than $W$ intersected with our trivializing chart. To see that, we choose a log resolution $\nu$ of the ideal $\left(f_{1}, \ldots, f_{p}\right)$ which produces on the resolution a holomorphic function 
$g$ and a trivial ideal sheaf $\left(g_{1}, \ldots, g_{p}\right)$ such that $\nu^{*} f_{i}=g_{i} g$ for each $i$. Therefore, $\nu^{*} \log H=$ $\log \sum\left|g_{i}\right|^{2}+\log |g|^{2}$, and the first summand extends smoothly across the exceptional divisor $(g=0)=\nu^{-1}(W)$.

Case 2. Near a point $x \in D \backslash W$, the hermitian metric $h$ is degenerate, but $\mathscr{F}$ is a genuine subbundle of $T_{X}(-\log D)$. A local holomorphic frame of $T_{X}(-\log D)$ is given by

$$
z_{1} \frac{\partial}{\partial z_{1}}, \ldots, z_{k} \frac{\partial}{\partial z_{k}}, \frac{\partial}{\partial z_{k+1}}, \ldots, \frac{\partial}{\partial z_{n}} .
$$

Also, $\omega$ is (possibly non-uniformly in $t, \varepsilon$, though) equivalent to the standard Kähler metric with cuspidal singularities along $D$. Therefore, in these coordinates $h$ is equivalent to the matrix $h_{\text {cusp }}:=\operatorname{diag}\left(\left(-\log \left|z_{1}\right|^{2}\right)^{-2}, \ldots,\left(-\log \left|z_{k}\right|^{2}\right)^{-2}, 1, \ldots, 1\right)$. Also, near $x$, the line bundle $\Lambda^{r} F$ is locally generated by one element $\xi \in \Lambda^{r} T_{X}(-\log D)$,

$$
\xi=\sum_{I=\left(i_{1}, \ldots, i_{r}\right)} a_{I}\left(\prod_{i \in I, i \leqslant k} z_{i}\right) \frac{\partial}{\partial z_{i_{1}}} \wedge \cdots \wedge \frac{\partial}{\partial z_{i_{r}}},
$$

where $I$ runs among all (unordered) $r$-tuples of $\{1, \ldots, n\}$. With respect to $\Lambda^{r} h_{\text {cusp }}$, the squared norm of $\xi$ is equal to $\sum_{I}\left|a_{I}\right|^{2} \prod_{i \in I \cap\{1, \ldots, k\}}\left(-\log \left|z_{i}\right|^{2}\right)^{-2}$. We choose an $r$-tuple $I$ such that $a_{I}(x) \neq 0$. One can assume that $a_{I}$ does not vanish, up to shrinking the neighborhood we are working on. As $h^{\wedge r}$ is equivalent to $\Lambda^{r} h_{\text {cusp }}$, we infer that

$$
\log |\xi|_{h^{\wedge r}}^{2} \geqslant \sum_{i \in I \cap\{1, \ldots, k\}}-\log \log ^{2}\left|z_{i}\right|^{2}-C
$$

for some given constant $C>0$. Remember that our ultimate goal is to show that

$$
\int_{X \backslash(W \cup D)} d d^{c} \log H \wedge \omega^{n-1}=0 .
$$

A way to prove this is to prove that $\log H$ is regular enough that this integral is actually cohomological. Of course, $\log H$ is not smooth (or even bounded), but the above inequality tends to suggest that it has finite energy in the sense of [GZ07]. But a zero-order bound like this does not quite suffice to support this claim, as we also crucially need to bound its complex Hessian from below. As $\xi$ is a (local) holomorphic section of the hermitian bundle $\left(\Lambda^{r} T_{X}, h\right)$ over $X \backslash D$, one can write

$$
d d^{c} \log |\xi|_{h}^{2}=\frac{1}{|\xi|_{h}^{2}}\left(|\nabla \xi|^{2}-\frac{|\langle\nabla \xi, \xi\rangle|^{2}}{|\xi|_{h}^{2}}\right)-\frac{\left\langle\Theta_{h}\left(\Lambda^{r} T_{X}\right) \xi, \xi\right\rangle}{|\xi|_{h}^{2}},
$$

where $\Theta_{h}\left(\Lambda^{r} T_{X}\right)$ is the Chern curvature tensor of $\left(\Lambda^{r} T_{X}, h\right)$ and $\nabla$ is the (1,0)-part of its Chern connection. As $|\langle\nabla \xi, \xi\rangle|^{2} \leqslant|\nabla \xi|^{2} \cdot|\xi|^{2}$, the term in the parentheses on the right-hand side is nonnegative. Moreover, we know from [KobR84, TY87] that $(X \backslash D, \omega)$ has bounded geometry, so in particular $\omega$ has bounded holomorphic bisectional curvature. Therefore the curvature tensor of $\left(\Lambda^{r} T_{X}, h\right)$ is bounded too, so that there is a constant $C$ (depending on $\varepsilon$ and $t$ ) such that $\Theta_{h}\left(\Lambda^{r} T_{X}\right) \leqslant C \omega \otimes \operatorname{Id}_{\Lambda^{r} T_{X}}$ in the sense of Griffiths semipositivity; in particular, we get

$$
d d^{c} \log |\xi|_{h}^{2} \geqslant-C \omega .
$$

Still, one cannot conclude yet that $\log H$ is in a finite energy class. Indeed, all we wrote requires $\xi$ not to vanish (so it applies as long as we are far away from $W$ ), and, also, the inequality (3.6) does not say that $\log H$ is quasi-plurisubharmonic (quasi-psh for short), as $\omega$ is certainly not dominated by a smooth Kähler form on $X$. The first issue will be addressed by using the methods of the first 


\section{H. Guenancia}

item above; as for the second, if we introduce $\varphi_{P}:=\sum-\log \log ^{2}\left|t_{j}\right|^{2}$, where the $t_{j}$ are sections cutting out the components of $D$ (and the norms are taken with respect to arbitrary smooth hermitian metrics), then $d d^{c} \varphi_{P}$ is quasi-psh and there exists a fixed smooth Kähler metric $\omega_{0}$ on $X$ such that $\omega_{0}+d d^{c} \varphi_{P}$ has cusp singularities along $D$. In particular, there exists a $C>0$ (depending on $t$ and $\varepsilon$, once again) such that $\omega_{0}+d d^{c} \varphi_{P} \geqslant C^{-1} \omega$. Also, it follows from [Gue14, Proposition 2.3] that $\varphi_{P} \in \mathcal{E}\left(X, \omega_{0}\right)$; that is, $\varphi_{P}$ has finite energy in the sense of [GZ07].

If $W$ were empty, then for some big constant $C>0$, the function $\log H+C \varphi_{P}$ would be a quasi-psh function dominating a multiple of $\varphi_{P}$ up to a constant, so it has finite energy too. Also, $\omega$ is a finite energy current (its potential is equal to $\left.\varphi_{P}+O(1)\right)$, therefore $\int_{X \backslash D} d d^{c}(\log H+$ $\left.C \varphi_{P}\right) \wedge \omega^{n-1}=0$. By linearity and as $\varphi_{P}$ has finite energy, we deduce $\int_{X \backslash D} d d^{c} \log H \wedge \omega^{n-1}=0$, which had to be showed.

Case 3. We now deal with the general case. The morphism $j: \operatorname{det} \mathscr{F} \rightarrow \Lambda^{r} T_{X}(-\log D)$ induces a non-zero section $t$ of $\Lambda^{r} T_{X}(-\log D) \otimes(\operatorname{det} \mathscr{F})^{-1}$. We choose a log resolution $\nu: \tilde{X} \rightarrow X$ of the ideal sheaf defined by $t$ and $D$, which is co-supported on $W \cup D$. Let $D^{\prime}$ be the strict transform of $D$, and let us analyze the behavior of $\nu^{*}(\log H)$ along $\operatorname{Exc}(\nu) \cup D^{\prime}$. By the previous two items, $\nu^{*}(\log H)$ dominates a (global) finite energy function locally outside $\operatorname{Exc}(\nu) \cap D^{\prime}$, and its complex Hessian is bounded from below by a (negative) constant times a cuspidal metric along $D^{\prime}$. So let us now see what happens at a point $x \in \operatorname{Exc}(\nu) \cap D^{\prime}$. Pick a small neighborhood $U$ of $x$, and let $\xi$ be a holomorphic trivialization of $\operatorname{det} \mathscr{F}$ on $U$. Over $U \backslash(W \cup D)$, the trivialization $\xi$ is a non-vanishing section of $\Lambda^{r} T_{X}(-\log D)$ that can be written

$$
\xi=\sum_{I=\left(i_{1}, \ldots, i_{r}\right)} a_{I}\left(\prod_{\substack{i \in I \\ i \leqslant k}} z_{i}\right) \frac{\partial}{\partial z_{i_{1}}} \wedge \cdots \wedge \frac{\partial}{\partial z_{i_{r}}}
$$

as above. Of course, the ideal sheaf defined by $t$ is given by $\left(a_{I}\right)$ on $U$. Moreover, as $\omega$ is equivalent to a cusp metric along $D$,

$$
\log |\xi|_{h}^{2}=\log \left(\sum_{I}\left|a_{I}\right|^{2} \prod_{i \in I \cap\{1, \ldots, k\}}\left(-\log \left|z_{i}\right|^{2}\right)^{-2}\right)+O(1) .
$$

We write $\nu^{*} a_{I}=g_{I} g$, where $g$ cuts out the exceptional divisor on $\nu^{-1}(U)$ and $\left(g_{I}\right)$ is a trivial ideal sheaf on this open set. Now, pick a point $\tilde{x}$ above $x$, that is, with $\nu(\tilde{x})=x$. There exist an $I$ such that $g_{I}(\tilde{x}) \neq 0$; moreover, as $\nu^{*} D$ is an snc divisor, there are holomorphic coordinates $\left(\tilde{z}_{1}, \ldots, \tilde{z}_{n}\right)$ around $\tilde{x}$ such that each of the $\nu^{*} z_{i}(1 \leqslant i \leqslant k)$ is a monomial in the $\tilde{z}_{j}$. Therefore, around $\tilde{x}$, we have

$$
\nu^{*} \log |\xi|_{h}^{2} \geqslant \log |g|^{2}+\sum_{i \in I \cap\{1, \ldots, k\}}-\log \log ^{2}\left|\nu^{*} z_{i}\right|^{2}-C .
$$

Take for example $\nu^{*} z_{1}$; by the observations above, we have $\nu^{*} z_{1}=\tilde{z}_{1}^{a_{1}} \cdots \tilde{z}_{n}^{a_{n}}$ for some nonnegative integers $a_{1}, \ldots, a_{n}$. Then $-\log \log ^{2}\left|\nu^{*} z_{i}\right|^{2}=-2 \log \left(-\sum_{i=1}^{n} a_{i} \log \left|\tilde{z}_{i}\right|^{2}\right)$, which by the concavity of the logarithm is greater than $\left(1 / \sum a_{i}\right) \sum_{i=1}^{n}-\log \log ^{2}\left|\tilde{z}_{i}\right|^{2}$. In the end, we obtain

$$
\nu^{*} \log |\xi|_{h}^{2} \geqslant \log |g|^{2}+k \sum_{i=1}^{n}-\log \log ^{2}\left|\tilde{z}_{i}\right|^{2}-C .
$$

Let us gather all that has been said so far. Take a section $\sigma$ cutting out the exceptional divisor $E$ of $\nu$, which we are going to measure with respect to an arbitrary smooth hermitian norm $h_{E}$. 


\section{SEMistability of THE TANGENT SHEAF OF SINGUlaR VARIETIES}

Let us set as before $\varphi_{P}:=\sum-\log \log ^{2}\left|s_{i}\right|^{2}$. We consider the function

$$
\psi:=\nu^{*}\left(\log \frac{|\xi|_{h}^{2}}{|\xi|_{h_{0}}^{2}}+C \varphi_{P}\right)-\log |\sigma|^{2}
$$

on $\nu^{-1}(X \backslash(W \cup D))$. We claim that for $C$ big enough, $\psi$ extends to a quasi-psh function on $\tilde{X}$ having finite energy. Indeed, by the bound $d d^{c} \log |\xi|_{h}^{2} \geqslant-C \omega$, there exists a Kähler metric $\omega_{0}$ on $X$ such that $d d^{c}\left(\log |\xi|_{h}^{2}+C \varphi_{P}\right) \geqslant-\omega_{0}$ outside $W \cup D$, for $C$ big enough. Therefore $d d^{c} \psi \geqslant-\tilde{\omega}_{0}$ on $\nu^{-1}(X \backslash(W \cup D))$ for some Kähler form $\tilde{\omega}_{0}$ on $\tilde{X}$. As $\psi$ is bounded from above thanks to (3.7), it therefore extends as a quasi-psh function on $\tilde{X}$. Moreover, we retrieve from (3.8) that near each point of the exceptional divisor (or $D^{\prime}$ ), $\psi$ dominates a function satisfying the criterion [BBGZ13, Lemma 2.9] thanks to [Gue14, Proposition 2.3], so $\psi$ satisfies this criterion globally, hence has finite energy. Therefore,

$$
\int_{\nu^{-1}(X \backslash(W \cup D))} d d^{c} \psi \wedge\left(\nu^{*} \omega\right)^{n-1}=0 .
$$

Moreover, $\varphi_{P}$ has finite energy, and the equality $d d^{c} \log |\sigma|^{2}=-\Theta_{h_{E}}(E)$ outside $E$ extends smoothly across the exceptional divisor (and $D$ ). As a result, we have

$$
\begin{aligned}
\int_{X \backslash(W \cup D)} d d^{c} \log H \wedge \omega^{n-1} & =\int_{\nu^{-1}(X \backslash(W \cup D))} \nu^{*} d d^{c} \log H \wedge\left(\nu^{*} \omega\right)^{n-1} \\
& =\int_{\nu^{-1}(X \backslash(W \cup D))} d d^{c}\left[\psi+\log |\sigma|^{2}-C \nu^{*} \varphi_{P}\right] \wedge\left(\nu^{*} \omega\right)^{n-1} \\
& =-\int_{\tilde{X}} \Theta_{h_{E}}(E) \wedge\left(\nu^{*} \omega\right)^{n-1},
\end{aligned}
$$

which vanishes as $E$ is $\nu$-exceptional and $\nu^{*} \omega$ is a finite energy current.

So we now know that we can use $h$ to compute the slope of $\mathscr{F}$. But outside $W \cup D$, the bundle $\mathscr{F}=\mathcal{O}_{X}(F)$ is a subbundle of $T_{X}$, so the Chern curvature tensors of each of the hermitian bundles can be related using the second fundamental form $\beta \in \Omega^{1,0} \otimes \operatorname{Hom}\left(F, F^{\perp}\right)$ :

$$
\Theta_{h}(F)=\operatorname{pr}_{F}\left(\Theta_{h}\left(T_{X}\right)_{\mid F}\right)+\beta^{*} \wedge \beta,
$$

where $\operatorname{pr}_{F}$ is the orthogonal projection onto $F$ of the endomorphism part. Taking the trace (as endomorphism), and then wedging with $\omega^{n-1}$, we get

$$
c_{1}(F, h) \wedge \omega^{n-1}=\operatorname{tr}_{\text {End }}\left(\operatorname{pr}_{F}\left(\operatorname{tr}_{\omega} \Theta_{h}\left(T_{X}\right)_{\mid F}\right)\right) \omega^{n} / n+\operatorname{tr}_{\text {End }}\left(\beta^{*} \wedge \beta \wedge \omega^{n-1}\right) .
$$

Integrating this identity over $X \backslash(W \cup D)$, we get, thanks to Proposition 3.8,

$$
n c_{1}(\mathscr{F}) \cdot\left(\pi^{*}\left(K_{Y}+\Delta\right)+t A\right)^{n-1} \leqslant \int_{X \backslash(W \cup D)} \operatorname{tr}_{\operatorname{End}}\left(\operatorname{pr}_{F}\left(\operatorname{tr}_{\omega} \Theta_{h}\left(T_{X}\right)_{\mid F}\right)\right) \omega^{n} .
$$

The next goal is then to compute the integral on the right-hand side and express it in terms of cohomological quantities.

Proposition 3.9. The integral

$$
\int_{X \backslash(W \cup D)} \operatorname{tr}_{\operatorname{End}}\left(\operatorname{pr}_{F}\left(\operatorname{tr}_{\omega} \Theta_{h}\left(T_{X}\right)_{\mid F}\right)\right) \omega^{n}
$$

converges to $-r\left(K_{Y}+\Delta\right)^{n}$ when first $\varepsilon$ and then $t$ converge to zero. 


\section{H. Guenancia}

Before starting the proof of the proposition, let us try understand what $\operatorname{tr}_{\omega} \Theta_{h}\left(T_{X}\right)$ is. We choose geodesic coordinates $\left(z_{i}\right)$ for $\omega$ around some point $x_{0}$, so that

$$
\Theta_{h}\left(T_{X}\right)_{x_{0}}=\sum_{j, k, l, m} R_{j \bar{k} l \bar{m}} d z_{j} \wedge d \bar{z}_{k} \otimes\left(\frac{\partial}{\partial z_{l}}\right)^{*} \otimes \frac{\partial}{\partial \bar{z}_{m}}
$$

In particular,

$$
\operatorname{tr}_{\omega} \Theta_{h}\left(T_{X}\right)_{x_{0}}=\sum_{j, l, m} R_{j \bar{j} l \bar{m}}\left(\frac{\partial}{\partial z_{l}}\right)^{*} \otimes \frac{\partial}{\partial \bar{z}_{m}}
$$

and using the Kähler symmetry $R_{j \bar{j} l \bar{m}}=R_{l \bar{m} j \bar{j}}$, we find

$$
\operatorname{tr}_{\omega} \Theta_{h}\left(T_{X}\right)_{x_{0}}=\sum_{j, l, m} R_{l \bar{m} j \bar{j}}\left(\frac{\partial}{\partial z_{l}}\right)^{*} \otimes \frac{\partial}{\partial \bar{z}_{m}} .
$$

It will be useful to introduce the operator $\sharp$ (relatively to $\omega$ ) which, with any $(1,1)$-form $\alpha$, associates an endomorphism $\sharp \alpha$ of $T_{X}$. More explicitly, if the metric $\omega$ is locally given by $\omega=$ $i \sum g_{\alpha \bar{\beta}} d z_{\alpha} \wedge d \bar{z}_{\beta}$ and if we denote by $\left(g^{\alpha \bar{\beta}}\right)$ the inverse matrix of $\left(g_{\alpha \bar{\beta}}\right)$, then $\sharp$ is defined by $\sharp d \bar{z}_{j}:=\sum_{k} g^{k \bar{j}} \frac{\partial}{\partial z_{k}}$. Therefore, if $\alpha=\sum_{i, j} \alpha_{i \bar{j}} d z_{i} \wedge d z_{\bar{j}}$, we have $\sharp \alpha=\sum_{i, j, k} \alpha_{i \bar{j}} g^{k \bar{j}} d z_{i} \otimes \frac{\partial}{\partial z_{k}}$. In particular,

$$
\operatorname{tr}_{\text {End }}(\sharp \alpha)=\operatorname{tr}_{\omega} \alpha \text {. }
$$

Recalling that $\operatorname{Ric} \omega=\sum_{j, l, m} R_{l \bar{m} j \bar{j}} d z_{l} \wedge d \bar{z}_{m}$, the computation above reads

$$
\operatorname{tr}_{\omega} \Theta_{h}\left(T_{X}\right)=\sharp(\operatorname{Ric} \omega) \text {. }
$$

We can now turn to the proof.

Proof of Proposition 3.9. Over $X \backslash D$, our metric $\omega$ satisfies equation (3.2):

$$
\operatorname{Ric} \omega=-\omega+t \omega_{A}-\sum_{a_{i}>-1} \theta_{i, \varepsilon}
$$

therefore $\operatorname{tr}_{\omega} \Theta_{h}\left(T_{X}\right)=-\sharp \omega+t \sharp \omega_{A}-\sum_{a_{i}>-1} \sharp \theta_{i, \varepsilon}$, and we have three terms to deal with.

First term. Of course, $\sharp \omega=\operatorname{Id}_{T_{X}}$, so that $\left.\operatorname{tr}_{\text {End }}\left(\operatorname{pr}_{F}(\sharp \omega)_{\mid F}\right)\right) \omega^{n}=r \omega^{n}$, which when integrated over $X \backslash(W \cup D)$ yields $r\left(\pi^{*}\left(K_{Y}+\Delta\right)+t A\right)^{n}$, as $\omega$ has finite energy. So our task is to show that as $\varepsilon$ and $t$ go to zero, the other terms vanish once properly contracted.

Second term. As for the second term, $\omega_{A}$ is a positive form, so $\sharp \omega_{A}$ is a positive endomorphism of $T_{X}$. Hence

$$
\operatorname{tr}_{\text {End }}\left(\operatorname{pr}_{F}\left(\left(\sharp \omega_{A}\right)_{\mid F}\right)\right) \omega^{n} \leqslant \operatorname{tr}\left(\sharp \omega_{A}\right) \omega^{n}=\operatorname{tr}_{\omega} \omega_{A} \omega^{n}=n \omega_{A} \wedge \omega^{n-1},
$$

and $t \int_{X \backslash(W \cup D)} n \omega_{A} \wedge \omega^{n-1}=\operatorname{tn} A \cdot\left(\pi^{*}\left(K_{Y}+\Delta\right)+t A\right)^{n-1}$ is independent of $\varepsilon$ and converges to zero when $t \rightarrow 0$. As a result, so does the non-negative integral $\int_{X \backslash(W \cup D)} \operatorname{tr}\left(\operatorname{pr}_{F}\left(\left(\sharp \omega_{A}\right)_{\mid F}\right)\right) \omega^{n}$.

Third term. The last term is the more subtle to deal with. We are going to show that for each $i$ such that $a_{i}>-1$, the integral $\left.\int_{X \backslash(W \cup D)} \operatorname{tr}_{\operatorname{End}}\left(\operatorname{pr}_{F}\left(\sharp \theta_{i, \varepsilon}\right)_{\mid F}\right)\right) \omega^{n}$ converges to zero. Remember that

$$
\theta_{i, \varepsilon}=a_{i}\left(\frac{\varepsilon^{2}\left|D^{\prime} s_{i}\right|^{2}}{\left(\left|s_{i}\right|^{2}+\varepsilon^{2}\right)^{2}}+\frac{\varepsilon^{2} \theta_{E_{i}}}{\left|s_{i}\right|^{2}+\varepsilon^{2}}\right)
$$

for the sake of clarity, let us drop the index $i$ and decompose $\theta$ as $\theta=a(\beta+\gamma)$, where

$$
\beta:=\frac{\varepsilon^{2}\left|D^{\prime} s\right|^{2}}{\left(|s|^{2}+\varepsilon^{2}\right)^{2}} \quad \text { and } \quad \gamma:=\frac{\varepsilon^{2} \theta_{E}}{|s|^{2}+\varepsilon^{2}} .
$$




\section{SEMistability of THE TANGENT SHEAF OF SINGUlaR VARIETIES}

Start with $\gamma$. One can choose $C>0$ big enough that $\pm \Theta \leqslant C \omega_{A}$. As the $\sharp$ operator respects positivity (and hence inequalities), we have

$$
\begin{aligned}
\left.\mid \operatorname{tr}_{\text {End }}\left(\operatorname{pr}_{F}(\sharp \gamma)_{\mid F}\right)\right) \mid \omega^{n} & \leqslant \frac{C \varepsilon^{2}}{|s|^{2}+\varepsilon^{2}} \operatorname{tr}\left(\operatorname{pr}_{F}\left(\left(\sharp \omega_{A}\right)_{\mid F}\right)\right) \omega^{n} \\
& \leqslant \frac{n C \varepsilon^{2}}{|s|^{2}+\varepsilon^{2}} \omega_{A} \wedge \omega^{n-1} ;
\end{aligned}
$$

hence

$$
\left.\mid \int_{X \backslash(W \cup D)} \operatorname{tr}_{\text {End }}\left(\operatorname{pr}_{F}(\sharp \gamma)_{\mid F}\right)\right) \omega^{n} \mid \leqslant C^{\prime} \int_{X \backslash(W \cup D)} \frac{\varepsilon^{2}}{|s|^{2}+\varepsilon^{2}} \omega_{A} \wedge \omega^{n-1},
$$

which converges to zero as $\varepsilon$ goes to zero, thanks to Lemma 3.7.

The term involving $\beta$ cannot be treated in the same way, as it explodes too quickly. However, we are going to take advantage of the facts that $\beta$ has a sign and that $\beta+\gamma$ belongs to a fixed contractible cohomological class. So similarly to what we had before, we have

$$
\left.\operatorname{tr}_{\operatorname{End}}\left(\operatorname{pr}_{F}(\sharp \beta)_{\mid F}\right)\right) \omega^{n} \leqslant n \beta \wedge \omega^{n-1} .
$$

Writing $\beta$ as $(\beta+\gamma)-\gamma$, we get

$$
\int_{X \backslash(W \cup D)} \operatorname{tr}\left(\operatorname{pr}_{F}\left((\sharp \beta)_{\mid F}\right)\right) \omega^{n} \leqslant C \int_{X \backslash(W \cup D)}\left(\theta \wedge \omega^{n-1}+\frac{\varepsilon^{2}}{|s|^{2}+\varepsilon^{2}} \omega_{A} \wedge \omega^{n-1}\right) .
$$

Using Lemma 3.7 and the fact that $\theta$ is smooth and $\omega$ has finite energy, we obtain that the right-hand side converges to

$$
C E \cdot\left(\pi^{*}\left(K_{Y}+\Delta\right)+t A\right)^{n-1}
$$

when $\varepsilon$ goes to zero, where $E$ is the ( $\pi$-exceptional) divisor representing the cohomology class of $\theta$. Therefore, our (non-negative) integral converges to zero as $t$ goes to zero, which ends the proof of Proposition 3.9.

Combining the inequality (3.10) with Proposition 3.9, we have shown that the slope of $\mathscr{F}$ is less than or equal to the slope of $T_{X}(-\log D)$, which concludes the proof of the semistability of $\mathscr{T}_{Y}(-\log \Delta)$ with respect to $K_{Y}+\Delta$.

Step 4. Polystability. Let us prove that the sheaf $\mathscr{T}_{Y}(-\log \Delta)$ is polystable with respect to $K_{Y}+\Delta$. Using an induction argument, it would be enough to prove that whenever there exists a sheaf $\mathscr{G} \subset \mathscr{T}_{Y}(-\log \Delta)$ with the same slope as $\mathscr{T}_{Y}(-\log \Delta)$, there also exists another sheaf $\mathscr{G}^{\prime}$ such that $\mathscr{T}_{Y}(-\log \Delta) \simeq \mathscr{G} \oplus \mathscr{G}^{\prime}$. Indeed, if $\mathscr{G}$ is chosen of minimal rank amongst the subsheaves of maximal slope, then $\mathscr{G}$ has to be stable, and we can run the whole argument with $\mathscr{G}^{\prime}$ instead of $\mathscr{T}_{Y}(-\log \Delta)$.

So let us assume the existence of such a subsheaf $\mathscr{G}$. By the arguments of step 1 , we can find a subsheaf $\mathscr{F}$ of $\mathscr{T}_{X}(-\log D)$ with the same slope as $\mathscr{T}_{X}(-\log D)$, which we may assume saturated (hence reflexive). Let us set $V=\pi^{-1}\left((Y, \Delta)_{\text {reg }}\right)=X \backslash \operatorname{Supp}(E)$ and $V_{0}:=V \backslash D=$ $X \backslash \operatorname{Supp}(D+E)$, and finally $U_{0}=V_{0} \backslash W$.

Going back to inequality (3.9), combining it with Proposition 3.9 and the fact that $\mathscr{F}$ has the same slope as $\mathscr{T}_{X}(-\log D)$, we obtain that the integral

$$
\int_{U_{0}} \operatorname{tr}_{\operatorname{End}}\left(\beta_{t, \varepsilon}^{*} \wedge \beta_{t, \varepsilon}\right) \wedge \omega_{t, \varepsilon}^{n-1}
$$




\section{H. Guenancia}

converges to zero when $\varepsilon$ and then $t$ go to zero. Here $\beta_{t, \varepsilon}$ is the second fundamental form of $F \subset T_{X}$ with respect to the hermitian metric induced by $\omega_{t, \varepsilon}$.

The crucial input we now need is in [BG14] (more precisely, Theorem 4.5 combined with the estimates from Sections 5.5 and 5.6: the estimates show that we can extract subsequences which will converge smoothly outside the singularities, and Theorem 4.5 says that all the limit metrics actually coincide and are equal to the Kähler-Einstein metric), where it is proved that there exists a Kähler metric $\omega_{\infty}$ on $V_{0}$ such that $\omega_{t, \varepsilon}$ converges to $\omega_{\infty}$ in the topology of $\mathscr{C}^{\infty}$ convergence on the compact subsets of $V_{0}$. Let $\beta_{\infty}$ the second fundamental form of $F \subset T_{X}$ induced by the Kähler metric $\omega_{\infty}$ on $U_{0}$. By the smooth convergence of $\omega_{t, \varepsilon}$ to $\omega_{\infty}$ on the compact subsets of $V_{0}$, Fatou's lemma applied to (3.12) shows that

$$
\int_{U_{0}} \operatorname{tr}\left(\beta_{\infty}^{*} \wedge \beta_{\infty}\right) \wedge \omega_{\infty}^{n-1}=0,
$$

so that $\operatorname{tr}\left(\beta_{\infty}^{*} \wedge \beta_{\infty}\right)$ and hence $\beta_{\infty}$ vanishes on $U_{0}$. In particular, we get an holomorphic splitting

$$
T_{X}(-\log D)_{\mid U_{0}} \simeq \mathscr{F}_{\mid U_{0}} \oplus \mathscr{F}_{\mid U_{0}}^{\perp} .
$$

We would like to extend this decomposition to $V=\pi^{-1}\left((Y, \Delta)_{\text {reg }}\right)$ and push it forward to

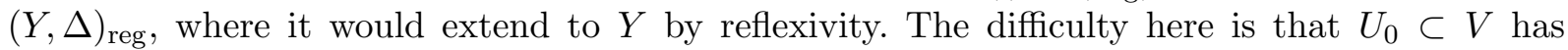
codimension 1 because of $D$. So we first have to show how extend the splitting across $D$ and then use reflexivity arguments to get it on $V$.

Another way to reformulate (3.13) is to say that there is a holomorphic surjection of vector bundles $p: T_{X}(-\log D) \rightarrow \mathscr{F}$ over $U_{0}$. Or better, we can view it as morphism $p: T_{X}(-\log D)$ $\rightarrow T_{X}(-\log D)$ over $U_{0}$ satisfying $p^{2}=p$ and with norm less that 1 ; that is, for every $v \in$ $T_{X}(-\log D)$, we have $|p(v)|_{\omega_{\infty}} \leqslant|v|_{\omega_{\infty}}$. We want to extend $p$ to $U \cap V$. The additional information we need lies in [GW16, Theorem B], where it is shown that $\omega_{\infty}$ is equivalent to a cusp metric along $D$ on $V$. Now we choose a point $x \in D \cap V$ such that $D$ is locally given by $D=\left(z_{1} \cdots z_{k}=0\right)$ for some coordinates $\left(z_{i}\right)$ around $x$. Then

$$
e_{i}:= \begin{cases}z_{i} \frac{\partial}{\partial z_{i}} & \text { if } 1 \leqslant i \leqslant k, \\ \frac{\partial}{\partial z_{i}} & \text { if } i>k\end{cases}
$$

defines a holomorphic frame of $T_{X}(-\log D)$ around $x$. By the previously cited result, the $\omega_{\infty^{-}}$ norm of $e_{i}$ is equivalent to $\left(\log \left(1 /\left|z_{i}\right|^{2}\right)\right)^{-1}$ if $1 \leqslant i \leqslant k$, and 1 if $i>k$. Let us consider $\left(p_{i j}(z)\right)$, the matrix of $p$ with respect to the basis $\left(e_{i}\right)$. We know that there exists a constant $C$ such that $\left|p\left(e_{i}\right)\right|_{\omega_{\infty}} \leqslant C$ for all $i$. One the other hand, as $\omega_{\infty}$ is equivalent to the cusp metric, we have

$$
\begin{aligned}
\left|p\left(e_{i}\right)\right|_{\omega_{\infty}}^{2} & =\left|\sum_{j=1}^{n} p_{i j}(z) e_{j}\right|_{\omega_{\infty}}^{2} \\
& \geqslant C^{-1}\left(\sum_{j=1}^{r} \frac{\left|p_{i j}(z)\right|^{2}}{\log ^{2} \frac{1}{\left|z_{i}\right|^{2}}}+\sum_{j=r+1}^{n}\left|p_{i j}(z)\right|^{2}\right) .
\end{aligned}
$$

Therefore, $\left|p_{i j}(z)\right|$ is uniformly bounded near $x$ if $j>k$, and $\left|p_{i j}(z)\right| \leqslant \log ^{2}\left(1 /\left|z_{i}\right|^{2}\right)$ if $1 \leqslant j \leqslant k$. In any case, $p_{i j}$ is locally $L^{2}$ near $x$ (with respect to a smooth volume form), and therefore it extends analytically across $D$. As a consequence, we get a holomorphic splitting

$$
\mathscr{T}_{X}(-\log D)_{\mid U \cap V} \simeq \mathscr{F}_{\mid U \cap V} \oplus \mathscr{F}_{\mid U \cap V}^{\perp}
$$


As the complement of $U \cap V$ in $V$ has codimension at least 2 and all the sheaves at play are reflexive, the above decomposition extends as a sheaf isomorphism

$$
\mathscr{T}_{X}(-\log D)_{\mid V} \simeq \mathscr{F}_{\mid V} \oplus\left(j_{U \cap V}\right)_{*}\left(\mathscr{F}_{\mid U \cap V}^{\perp}\right),
$$

where $j_{U \cap V}: U \cap V \rightarrow V$ is the open immersion. Pushing forward by $\pi$, we get on $(Y, \Delta)_{\text {reg: }}$ :

$$
\mathscr{T}_{Y}(-\log \Delta)_{\mid(Y, \Delta)_{\mathrm{reg}}} \simeq \mathscr{G}_{\mid(Y, \Delta)_{\mathrm{reg}}} \oplus \mathscr{G}_{\mid(Y, \Delta)_{\mathrm{reg}}^{\prime}}^{\prime},
$$

where $\mathscr{G}^{\prime}$ is the (reflexive) sheaf on $Y$ defined as $\left(j_{(Y, \Delta)_{\text {reg }}} \circ \pi \circ j_{U \cap V}\right)_{*}(\mathscr{F} \stackrel{\perp}{\mid U \cap V})$, where $j_{\mid}: \cdot \rightarrow Y$ generically denotes any open immersion from - to $Y$. And here again, the reflexivity of these sheaves leads to the expected splitting

$$
\mathscr{T}_{Y}(-\log \Delta) \simeq \mathscr{G} \oplus \mathscr{G}^{\prime}
$$

on $Y$, ending the proof of the polystability of $\mathscr{T}_{Y}(-\log \Delta)$.

Remark 3.10. We already mentioned in the introduction and in Section 3.2 that Theorem 3.5 is a significant generalization of what was known before, that is, the semistability of the tangent sheaf in the case of canonical singularities; cf. [Eno88]. However, two consequential simplifications occur if one is interested in a weakened form of Theorem 3.5.

First, if one focuses only on semistability and not polystability; then, instead of using the inequality relating the Chern curvature form of a subbundle $F$ to that of the ambient bundle $T_{X}$ (cf. (3.9)), one can use a weaker form of it as in [CP14, equation (1.2)] which enables us to endow $\operatorname{det} F$ with a smooth metric instead of the singular metric induced by $\omega$ (the singularities occur because $F$ is a subbundle only on an open set, and because $\omega$ may have cuspidal singularities along a divisor), thus avoiding the use of the technical Lemma 3.8.

Second, if $\Delta=0$, then the approximate Kähler-Einstein metrics $\omega$ are smooth and do not have cuspidal singularities anymore. In that case, the crucial Lemma 3.8 becomes much easier and was already treated in the item $(* *)$ of the proof of [KobS87, Theorem 8.3].

\section{An extension to stable varieties}

\subsection{Stable varieties}

Once we have studied log canonical pairs with ample log canonical bundle, it is very tempting to try to extend these results to varieties (or pairs) with possible non-normal singularities. More precisely, we will consider stable varieties in the sense of Kollár-Shepherd-Barron [KSB88] and Alexeev [Ale96]. These varieties are the higher-dimensional analogue of stable curves as defined by Deligne-Mumford [DM69], and they arise in the compactification of the moduli space of smooth canonically polarized projective varieties; their precise definition is stated below, though we refer to the very nice paper [Kov13] and the references therein for more details and insight on all the objects involved.

Definition 4.1. A stable variety is an equidimensional and reduced complex projective variety $X$ with semi-log canonical singularities such that $K_{X}$ is ample.

Let us recall that $X$ has semi-log canonical singularities if the only singularities of $X$ in codimension 1 are ordinary double points (that is, locally analytically isomorphic to $(x y=0) \in$ $\left.\mathbb{C}^{n+1}\right)$, if $X$ satisfies Serre's $S_{2}$ property and is $\mathbb{Q}$-Gorenstein, and if the pair $\left(X^{\nu}, D^{\nu}\right)$ formed by the normalization of $X$ and its conductor divisor has log canonical singularities. Here the 


\section{H. Guenancia}

conductor of the normalization is essentially the Weil divisor determined by the preimage of the ordinary double points locus under the normalization map.

It is important to notice that stable varieties may be reducible, which is a source of difficulties in view of the theory of semistability for coherent sheaves. Indeed, if we want to mimic the definition of the slope of a coherent sheaf $\mathscr{F}$, we need to define the $(n-1)$-cycle $c_{1}(\mathscr{F})$. We can still do it on $X_{\mathrm{reg}}$, but there is an issue when we want to extend it to the whole $X$, as the complement of $X_{\text {reg }}$ has codimension 1 in general. Actually, there is a very general notion of slope, defined in a high degree of generality using Hilbert polynomials (see, for example, [HL10]), and of course agreeing with the usual one on smooth varieties. We are now going to review these notions briefly.

\subsection{Slope and stability on non-normal varieties}

On non-normal varieties, defining the slope of a coherent sheaf is more complicated. We recall the definition; cf. [HL10]. Let $X$ be an $n$-equidimensional reduced projective scheme over $\mathbb{C}$, let $L$ be an ample line bundle, and let $\mathscr{F}$ be a coherent sheaf on $X$ of dimension $n$ (which means that the support of $\mathscr{F}$ has dimension $n)$. We know that the Hilbert polynomial $P(\mathscr{F})$ given by $m \mapsto \chi\left(X, \mathscr{F} \otimes L^{\otimes m}\right)$ can be uniquely written in the form

$$
P(\mathscr{F}, m):=\sum_{k=0}^{n} a_{k}(\mathscr{F}) \frac{m^{k}}{k !},
$$

where the $a_{k}(\mathscr{F})$ are integers. Note that for $m$ large enough, the vanishing of the higher cohomology implies $P(\mathscr{F}, m)=h^{0}\left(X, \mathscr{F} \otimes L^{\otimes m}\right)$. Of course, this polynomial depends on $\mathscr{F}$ and on the polarization $L$. If $X$ is integral, then the rank of $\mathscr{F}$ is defined as the rank of $\mathscr{F}$ at the generic point (as $\mathscr{F}$ is locally free on some dense open set). In this more general framework, we define the rank of $\mathscr{F}$ as a convex combination of the ranks of $\mathscr{F}$ restricted to each irreducible component. More precisely, if $X=X_{1} \cup \cdots \cup X_{r}$, where the $X_{i}$ are the irreducible components of $X$, and if $L_{i}$ and $\mathscr{F}_{i}$ denote the restrictions of $L$ and $\mathscr{F}$ to $X_{i}$, respectively, then we define

$$
\operatorname{rk}(\mathscr{F}):=\frac{\sum_{i=1}^{r}\left(L_{i}^{n}\right) \operatorname{rk}\left(\mathscr{F}_{i}\right)}{\left(L^{n}\right)} .
$$

One can also check that $\operatorname{rk}(\mathscr{F})$ coincides with $a_{n}(\mathscr{F}) / a_{n}\left(\mathcal{O}_{X}\right)$; cf. [Laz04, Remark 1.1.26]. If $\mathscr{F}$ has constant rank (that is, $\operatorname{rk}\left(\mathscr{F}_{i}\right)$ is independent of $\left.i\right)$, then the rank of $\mathscr{F}$ is the same $\operatorname{as} \operatorname{rk}\left(\mathscr{F}_{i}\right)$ for any $i$.

Now we need to define the degree of $\mathscr{F}$ with respect to $L$. To do this, we introduce the suitable combination of the coefficients $a_{k}$ that gives the usual degree in the smooth case. More precisely,

$$
\operatorname{deg}_{L}(\mathscr{F}):=a_{n-1}(\mathscr{F})-\operatorname{rk}(\mathscr{F}) a_{n-1}\left(\mathcal{O}_{X}\right),
$$

and finally, one can define the slope as the quotient of the degree by the rank,

$$
\mu_{L}(\mathscr{F}):=\frac{\operatorname{deg}_{L}(\mathscr{F})}{\operatorname{rk}(\mathscr{F})} .
$$

Of course, this definition only make sense for sheaves with positive rank.

Remark 4.2. If $X=X_{1} \amalg \cdots \amalg X_{s}$ is the disjoint sum of its irreducible components, and if $\mathscr{F}$ has constant rank, then it follows from the definition that the slope of $\mathscr{F}$ with respect to a polarization $L$ on $X$ is the sum of the slopes of $\mathscr{F}_{\mid X_{i}}$ with respect to $L_{\mid X_{i}}$ for $1 \leqslant i \leqslant s$. 
The first thing to check is whether this definition generalizes the definition of slope that we gave for normal varieties. Recall that if $\mathscr{F}$ is a coherent sheaf on a normal variety, we defined $c_{1}(\mathscr{F})$ to be the $(n-1)$-cycle represented by the closure of the Weil divisor attached to $\operatorname{det}\left(\mathscr{F}_{\mid X_{\text {reg }}}\right)$.

Proposition 4.3. Let $(X, L)$ be a polarized normal variety of dimension $n$ and $\mathscr{F}$ a torsion-free coherent sheaf on $X$. Then we have

$$
\operatorname{deg}_{L}(\mathscr{F})=\left(c_{1}(\mathscr{F}) \cdot L^{n-1}\right),
$$

and, in particular, $\mu_{L}(\mathscr{F})$ agrees with $\left(c_{1}(\mathscr{F}) \cdot L^{n-1}\right) / \operatorname{rk}(\mathscr{F})$.

Proof. Let $\pi: Y \rightarrow X$ be a resolution of $X$ such that $\pi$ is an isomorphism over $X_{\text {reg. We will }}$ show that the two quantities above coincide with $\left(c_{1}\left(\pi^{*} \mathscr{F}\right) \cdot \pi^{*} L^{n-1}\right)$.

Using the projection formula and the fact that on $X_{\text {reg }}$, the cycles $\pi_{*} c_{1}\left(\pi^{*}(\mathscr{F})\right)$ and $c_{1}(\mathscr{F})$ coincide, we see that $\left(c_{1}\left(\pi^{*} \mathscr{F}\right) \cdot \pi^{*} L^{n-1}\right)=\left(c_{1}(\mathscr{F}) \cdot L^{n-1}\right)$, so we have done half of the job. The other equality requires a bit more work.

First, let us prove that $\operatorname{deg}_{L}(\mathscr{F})=\operatorname{deg}_{\pi^{*} L}\left(\pi^{*} \mathscr{F}\right)$. If we define $\mathscr{G}$ to be the sheaf $\pi^{*} \mathscr{F} \otimes \pi^{*} L^{\otimes m}$, then the degeneracy of the Leray spectral sequence provides the equality

$$
\chi(Y, \mathscr{G})=\sum_{q \geqslant 0}(-1)^{q} \chi\left(X, R^{q} \pi_{*} \mathscr{G}\right) .
$$

Moreover, the projection formula gives us an isomorphism

$$
R^{q} \pi_{*}\left(\pi^{*} \mathscr{F} \otimes_{\mathcal{O}_{Y}} \pi^{*} L^{\otimes m}\right) \rightarrow R^{q} \pi_{*}\left(\pi^{*} \mathscr{F}\right) \otimes_{\mathcal{O}_{X}} L^{\otimes m} .
$$

As $\mathscr{F}$ is torsion free, it is locally free in codimension 1 (cf., for example, [KobS87, 5.15]), so if we apply the projection formula once again, we obtain that the natural morphism

$$
R^{q} \pi_{*}\left(\pi^{*} \mathscr{F}\right) \rightarrow R^{q} \pi_{*} \mathcal{O}_{Y} \otimes_{\mathcal{O}_{X}} \mathscr{F}
$$

is an isomorphism in codimension 2. Combining these two observations, we get a morphism

$$
R^{q} \pi_{*}\left(\pi^{*} \mathscr{F} \otimes_{\mathcal{O}_{Y}} \pi^{*} L^{\otimes m}\right) \rightarrow R^{q} \pi_{*} \mathcal{O}_{Y} \otimes_{\mathcal{O}_{X}} \mathscr{F} \otimes_{\mathcal{O}_{X}} L^{\otimes m},
$$

which is an isomorphism in codimension 2. Therefore, as $\pi_{*} \mathcal{O}_{Y}=\mathcal{O}_{X}$, we get from (4.1):

$$
\chi\left(Y, \pi^{*} \mathscr{F} \otimes \pi^{*} L^{\otimes m}\right)=\chi\left(X, \mathscr{F} \otimes L^{\otimes m}\right)+\sum_{q>0}(-1)^{q} \chi\left(X, R^{q} \pi_{*} \mathcal{O}_{Y} \otimes \mathscr{F} \otimes L^{\otimes m}\right)+O\left(m^{n-2}\right) .
$$

Finally, as $X$ is normal and $\pi$ is an isomorphism over $X_{\text {reg }}$, the sheaves $R^{q} \pi_{*} \mathcal{O}_{Y}$ are supported in codimension at most 2 for $q>0$ (cf. [Har77, 8.2 and 11.2], for example). Therefore, we obtain

$$
\chi\left(Y, \pi^{*} \mathscr{F} \otimes \pi^{*} L^{\otimes m}\right)=\chi\left(X, \mathscr{F} \otimes L^{\otimes m}\right)+O\left(m^{n-2}\right) .
$$

These two polynomials have same leading-term coefficient, namely the top intersection of $c_{1}(\mathscr{F}) \otimes$ $L$ divided by $n$ ! (as $\pi$ is generically of degree 1 ), so they have the same coefficient in front of the second-highest power of $m$. As this is true for any torsion-free sheaf $\mathscr{F}$, we have the same result for $\mathcal{O}_{X}$ too, so in the end we get $\operatorname{deg}_{L}(\mathscr{F})=\operatorname{deg}_{\pi^{*} L}\left(\pi^{*} \mathscr{F}\right)$.

To conclude the proof of the proposition, we need to see that $\operatorname{deg}_{\pi^{*} L}\left(\pi^{*} \mathscr{F}\right)=\left(c_{1}\left(\pi^{*} \mathscr{F}\right)\right.$. $\left.\pi^{*} L^{n-1}\right)$. By the Riemann-Roch-Hirzebruch theorem, we know that

$$
\begin{aligned}
\chi\left(Y, \pi^{*} \mathscr{F} \otimes \pi^{*} L^{\otimes m}\right)= & \operatorname{rk}(\mathscr{F}) \cdot\left(\pi^{*} L\right)^{n} \frac{m^{n}}{n !} \\
& +\left[\left(c_{1}\left(\pi^{*} \mathscr{F}\right) \cdot \pi^{*} L^{n-1}\right)+\frac{1}{2}\left(c_{1}(Y) \cdot \pi^{*} L^{n-1}\right)\right] \frac{m^{n-1}}{(n-1) !}+O\left(m^{n-2}\right) .
\end{aligned}
$$

Applying this to $\mathscr{F}$ and $\mathcal{O}_{Y}$, we get the expected equality. 


\section{H. Guenancia}

Now we would like to see, for a non-normal variety, how to relate the slope of a sheaf with the slope of its pull-back by the normalization map. This is the content of the following proposition.

Proposition 4.4. Let $X$ be a reduced equidimensional projective scheme over $\mathbb{C}$, let $L$ be a polarization, and let $\mathscr{F}$ be a coherent sheaf which is locally free in codimension 1. If $\pi: Y \rightarrow X$ is the normalization of $X$, then we have

$$
\mu_{\pi^{*} L}\left(\pi^{*} \mathscr{F}\right)=\mu_{L}(\mathscr{F}) .
$$

Proof. First, let us define $\mathcal{T}:=\pi_{*} \mathcal{O}_{Y} / \mathcal{O}_{X}$, and let us set $r=\operatorname{rk}(\mathscr{F})$ (one may notice that $\mathscr{F}$, being locally free in codimension 1 , has constant rank). As $L$ and $\pi^{*} L$ are ample, we will assume in the following that the power $m$ to which we raise them is large enough that all the higher cohomology groups involved are zero. As a consequence, we will identify the Euler-Poincaré characteristic with $h^{0}$. Using the projection formula combined with an argument similar to that in the previous proof (we use here that $\mathscr{F}$ is locally free in codimension 1), we see that

$$
h^{0}\left(Y, \pi^{*} \mathscr{F} \otimes \pi^{*} L^{\otimes m}\right)=h^{0}\left(X, \mathscr{F} \otimes L^{\otimes m} \otimes \pi_{*} \mathcal{O}_{Y}\right)+O\left(m^{n-2}\right) .
$$

Moreover, as $\mathscr{F}$ is locally free in codimension 1 , the short sequence

$$
0 \rightarrow \mathscr{F} \otimes L^{\otimes m} \rightarrow \mathscr{F} \otimes L^{\otimes m} \otimes \pi_{*} \mathcal{O}_{Y} \rightarrow \mathscr{F} \otimes L^{\otimes m} \otimes \mathcal{T} \rightarrow 0
$$

is exact in codimension 1. Combining these two identities, we get

$$
a_{n-1}\left(\pi^{*} \mathscr{F}\right)=a_{n-1}(\mathscr{F})+a_{n-1}(\mathscr{F} \otimes \mathcal{T}),
$$

where the polarizations which respect to which the Hilbert polynomials are computed are respectively $L$ on $X$ and $\pi^{*} L$ on $Y$.

Therefore, we have

$$
\begin{aligned}
\mu_{\pi^{*} L}\left(\pi^{*} \mathscr{F}\right) & =\frac{1}{r} a_{n-1}\left(\pi^{*} \mathscr{F}\right)-a_{n-1}\left(\mathcal{O}_{Y}\right) \\
& =\frac{1}{r} a_{n-1}(\mathscr{F})+\frac{1}{r} a_{n-1}(\mathscr{F} \otimes \mathcal{T})-a_{n-1}\left(\mathcal{O}_{X}\right)+\left(a_{n-1}\left(\mathcal{O}_{Y}\right)-a_{n-1}\left(\mathcal{O}_{X}\right)\right) \\
& =\mu_{L}(\mathscr{F})+\frac{1}{r} a_{n-1}(\mathscr{F} \otimes \mathcal{T})-a_{n-1}(\mathcal{T}) .
\end{aligned}
$$

If $\mathcal{T}$ is supported in codimension at most 2 , then $\frac{1}{r} a_{n-1}(\mathscr{F} \otimes \mathcal{T})=a_{n-1}(\mathcal{T})=0$ and we are done. Else, it is supported in a codimension 1 subvariety $Z$, so it follows from the Riemann-Roch formula (in the reducible case, cf. [Kol96, Proposition VI.2.7]) that $a_{n-1}(\mathscr{F} \otimes \mathcal{T})=\operatorname{rk}((\mathscr{F} \otimes$ $\left.\mathcal{T})_{\mid Z}\right)=r \cdot \operatorname{rk}(\mathcal{T})=r a_{n-1}(\mathcal{T})$, where we again used that $\mathscr{F}$ is locally free in codimension 1 . In the end, we have proved that the slopes of $\mathscr{F}$ and $\pi^{*} \mathscr{F}$ agree.

EXAMPLE 4.5. One should pay attention to the fact that the above result is false without the assumption that the sheaf is locally free in codimension 1 . Indeed, if we choose $X=(x y=0) \subset \mathbb{P}^{2}$ to be the union of two lines, then its normalization is $\mathbb{P}^{1}$ with two points $p^{\prime}$ and $p^{\prime \prime}$ sitting above the node $p$. Then, if we choose $L=\mathcal{O}_{\mathbb{P}^{2}}(1)_{\mid X}$ and $\mathscr{F}=\mathcal{O}_{X} \oplus\left(\mathcal{O}_{X} / \mathcal{I}_{p}\right)$, where $I$ is the ideal sheaf of the node, we see that $h^{0}\left(\mathscr{F} \otimes L^{\otimes m}\right)=h^{0}\left(L^{\otimes m}\right)+1$; that is, $\mu_{L}(\mathscr{F})=1$. But $\pi^{*} \mathscr{F}=\mathcal{O}_{Y} \oplus \mathcal{O}_{Y} / \mathcal{I}_{p^{\prime}, p^{\prime \prime}}$, so that $h^{0}\left(\pi^{*} \mathscr{F} \otimes \pi^{*} L^{\otimes m}\right)=h^{0}\left(\pi^{*} L^{\otimes m}\right)+2$ and therefore $\mu_{\pi^{*} L}\left(\pi^{*} \mathscr{F}\right)=2$.

In view of all the observations we have made so far, one can introduce a notion of semistability which is weaker than the one in [HL10, 1.2.12], but particularly adapted to our context.

DeFinition 4.6. Let $X$ be an $n$-equidimensional reduced projective scheme over $\mathbb{C}$ and $L$ an ample divisor. A coherent sheaf $\mathscr{E}$ is said to be semistable with respect to $L$ if for every subsheaf 
$\mathscr{F} \subset \mathscr{E}$ that is locally free in codimension 1 and satisfies $0<\operatorname{rk}(\mathscr{F})<\operatorname{rk}(\mathscr{E})$, we have $\mu_{L}(\mathscr{F}) \leqslant$ $\mu_{L}(\mathscr{E})$.

If $X$ is normal and $\mathscr{E}$ is torsion free, then any subsheaf $\mathscr{F}$ of $\mathscr{E}$ is also torsion free, hence locally free in codimension 1 , as $X$ is normal. So we just recover the usual notion of semistability.

\subsection{Main result}

The general definition above is actually motivated by Proposition 4.4 and tailored for the following theorem.

TheOREM 4.7. Let $X$ be a stable variety, let $\nu: X^{\nu} \rightarrow X$ be the normalization of $X$, and let $\Delta$ be the conductor of $\nu$. Then the sheaf $\nu_{*} \mathscr{T}_{X^{\nu}}(-\log \Delta)$ is semistable with respect to $K_{X}$.

Although stability has been defined with respect to a genuine ample line bundle, one can work as well with $\mathbb{Q}$-line polarizations $L$. To do so, one considers $m_{0}$ such that $m_{0} L$ is Cartier and shows stability with respect to $m_{0} L$ independently of the choice of such an integer $m_{0}$.

Proof. Let $\mathscr{F} \subset \mathscr{T}_{X}$ be a coherent subsheaf of $\mathscr{T}_{X}$ which is locally free in codimension 1. If $\nu: X^{\nu} \rightarrow X$ is the normalization of $X$ and $\Delta$ is the conductor divisor of $\nu$ on $X^{\nu}$, then we get a generically injective map $\nu^{*} \mathscr{F} \rightarrow \nu^{*} \nu_{*} \mathscr{T}_{X^{\nu}}(-\log \Delta)$. If we compose it with the natural map $\nu^{*} \mathscr{T}_{X} \rightarrow\left(\nu^{*} \nu_{*} \mathscr{T}_{X^{\nu}}(-\log \Delta)\right)^{* *}$, then in view of the Lemma 4.9 below, we get a generically injective morphism

$$
\nu^{*} \mathscr{F} \rightarrow \mathscr{T}_{X^{\nu}}(-\log \Delta) .
$$

Let us now consider a log resolution $\pi: Y \rightarrow X^{\nu}$ of $\left(X^{\nu}, \Delta\right)$ that leaves the snc locus untouched, and let $D=\pi^{-1}(\Delta)$. Then there is a natural morphism $\pi^{*} \mathscr{T}_{X^{\nu}}(-\log \Delta) \rightarrow T_{Y}(-\log D)$ which is

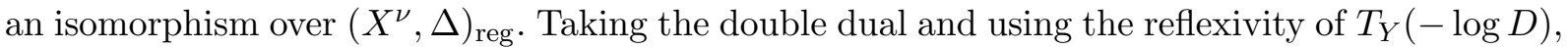
we end up with a natural isomorphism $\left(\pi^{*} T_{X^{\nu}}(-\log \Delta)\right)^{* *} \simeq T_{Y}(-\log D)$. Pulling back (4.2) by $\pi$, we get a generically injective morphism $\pi^{*} \nu^{*} \mathscr{F} \rightarrow T_{Y}(-\log D)$. We proved that in this situation, the slope of $\pi^{*} \nu^{*} \mathscr{F}$ with respect to $\pi^{*} \nu^{*} K_{X}$ is less than or equal to the slope of $T_{Y}(-\log D)$. At this point, we use the fact that $\mathscr{F}$ has constant rank to pass from the slope inequality on each component to the global slope inequality.

By the projection formula, it yields

$$
\mu_{\nu^{*} K_{X}}\left(\nu^{*} \mathscr{F}\right) \leqslant \mu_{\nu^{*} K_{X}}\left(\mathscr{T}_{X^{\nu}}(-\log \Delta)\right) .
$$

We would like to push forward this inequality to $X$. For the left-hand side, this can be done thanks to Proposition 4.4, which shows that $\mu_{\nu^{*} K_{X}}\left(\nu^{*} \mathscr{F}\right)=\mu_{K_{X}}(\mathscr{F})$ as $\mathscr{F}$ is locally free in codimension 1. Actually, the right-hand side is not equal to $\mu_{K_{X}}\left(\mathscr{T}_{X}\right)$, but there is a correction factor which fortunately has the right sign. More precisely, writing $\mathcal{T}:=\nu_{*} \mathcal{O}_{X^{\nu}} / \mathcal{O}_{X}$, by the projection formula ( $m$ is large and divisible enough), we have

$$
h^{0}\left(X^{\nu}, \nu^{*}\left(K_{X}^{\otimes m}\right)^{* *}\right)=h^{0}\left(X,\left(K_{X}^{\otimes m}\right)^{* *} \otimes \nu_{*} \mathcal{O}_{X^{\nu}}\right)
$$

and also

$$
h^{0}\left(X^{\nu}, \mathscr{T}_{X^{\nu}}(-\log \Delta) \otimes \nu^{*}\left(K_{X}^{\otimes m}\right)^{* *}\right)=h^{0}\left(X, \nu_{*} \mathscr{T}_{X^{\nu}}(-\log \Delta) \otimes\left(K_{X}^{\otimes m}\right)^{* *}\right) .
$$




\section{H. Guenancia}

Therefore, we get

$$
\begin{aligned}
\mu_{\nu^{*} K_{X}}\left(\mathscr{T}_{X^{\nu}}(-\log \Delta)\right) & =\frac{1}{n} a_{n-1}\left(\mathscr{T}_{X^{\nu}}(-\log \Delta)\right)-a_{n-1}\left(\mathcal{O}_{X^{\nu}}\right) \\
& =\left(\frac{1}{n} a_{n-1}\left(\nu_{*} \mathscr{T}_{X^{\nu}}(-\log \Delta)\right)-a_{n-1}\left(\mathcal{O}_{X}\right)\right)+a_{n-1}\left(\mathcal{O}_{X}\right)-a_{n-1}\left(\mathcal{O}_{X^{\nu}}\right) \\
& =\mu_{K_{X}}\left(\nu_{*} \mathscr{T}_{X^{\nu}}(-\log \Delta)\right)-a_{n-1}(\mathcal{T}) .
\end{aligned}
$$

As $\mathcal{T}$ is supported in codimension at least 1 , we have $a_{n-1}(\mathcal{T}) \geqslant 0$, so that in the end, we have proved

$$
\mu_{K_{X}}(\mathscr{F}) \leqslant \mu_{K_{X}}\left(\nu_{*} \mathscr{T}_{X^{\nu}}(-\log \Delta)\right) .
$$

Remark 4.8. In general, the sheaf $\nu_{*} \mathscr{T}_{X^{\nu}}(-\log \Delta)$ is distinct from the tangent sheaf of $X$ (defined as the sheaf of derivations of $\mathcal{O}_{X}$ ), as already in the case of a (local) node $X=$ $\operatorname{Spec}(\mathbb{C}[x, y, z] /(x y))$, the former sheaf strictly contains the latter one. But given the last step of the proof, where we neglected $a_{n-1}(T)$, it could be possible that the same proof yields the semistability of $\mathscr{T}_{X}$.

To conclude this section, let us state and prove a result we have used in the course of the proof of Theorem 4.7.

Lemma 4.9. Let $X$ be an $n$-equidimensional reduced projective scheme over $\mathbb{C}$, and let $\pi: Y \rightarrow X$ be the normalization of $X$. If $\mathscr{E}$ is a reflexive coherent sheaf on $Y$, then the reflexive hull $\left(\pi^{*} \pi_{*} \mathscr{E}\right)^{* *}$ is naturally isomorphic to $\mathscr{E}$.

Proof. Let us begin by reducing the problem to one of commutative algebra. First, there is a natural map $\pi^{*} \pi_{*} \mathscr{E} \rightarrow \mathscr{E}$ inducing $\left(\pi^{*} \pi_{*} \mathscr{E}\right)^{* *} \rightarrow \mathscr{E}^{* *} \simeq \mathscr{E}$. This map is a morphism between two reflexive sheaves, so if we can show that it is a isomorphism over a open set in $Y$ whose complement has codimension at least 2 , then our map will actually be a sheaf isomorphism over the whole $Y$. As a consequence, one can assume that $\mathscr{E}$ is locally free (as $\mathscr{E}$ is reflexive, it will be locally free on such a "big" open set). Now, the problem is local, and as the normalization is an affine morphism, one can assume without loss of generality that $Y=\operatorname{Spec}(B), X=\operatorname{Spec}(A)$ and $\mathscr{E}=\widetilde{B}$. We claim that the sheaf morphism $\mathscr{E}^{*} \rightarrow\left(\pi^{*} \pi_{*} \mathscr{E}\right)^{*}$ is an isomorphism, or equivalently that the morphism of $B$-modules

$$
\begin{aligned}
F: \operatorname{Hom}_{B}(B, B) & \rightarrow \operatorname{Hom}_{B}\left(B \otimes_{A} B_{A}, B\right) \\
\varphi & \longmapsto \varphi \circ f
\end{aligned}
$$

is an isomorphism. Here, $B_{A}$ is $B$ viewed as an $A$-module and $f: B \otimes_{A} B_{A} \rightarrow B$ is defined by $f\left(\sum b_{i} \otimes m_{i}\right)=\sum b_{i} m_{i}$. As $f$ is surjective, $F$ is injective, so we need to prove that $F$ is surjective. Given $\psi \in \operatorname{Hom}_{B}\left(B \otimes B_{A}, B\right)$, we define $\varphi \in \operatorname{Hom}_{B}(B, B)$ by setting $\varphi(1)=\psi(1 \otimes 1)$. We want to show that $F(\varphi)=\psi$, and this amounts to proving that for any $b \in B$, we have $\psi(1 \otimes b)=b \psi(1 \otimes 1)$. The crucial input is that $B$ is a subring of the total quotient ring of $A$, so there exists an $s \in A$ which is not zero divisor, such that $s b \in A$. Therefore, if we set $x:=\psi(1 \otimes b)-b \psi(1 \otimes 1)$, we get $s x=s \psi(1 \otimes b)-s b \psi(1 \otimes 1)=\psi(s \otimes b)-\psi(s b \otimes 1)=\psi(1 \otimes s b)-\psi(s b \otimes 1)=0$, as $s b \in A$. So $s x=0$; but $s$ is not a zero divisor in $A$ and hence neither in $B$, as $B$ is a subring of the total quotient ring of $A$. This proves $x=0$, and thus $F$ is an isomorphism, as claimed. 


\section{Generic semipositivity}

In this section, we prove Theorem C, stated in the introduction. Recall that a reflexive sheaf $\mathscr{E}$ on a normal compact Kähler space $X$ endowed with a Kähler form $\omega$ is said to be generically $\omega$ semipositive if for all coherent quotient $\mathscr{F}$ of $\mathscr{E}$, the degree of $\mathscr{F}$ with respect to $\omega$ is non-negative, that is, $\int_{X} c_{1}(\mathscr{F}) \wedge \omega^{n-1} \geqslant 0$.

By a theorem of Hartshorne [Har71], a vector bundle over a curve is nef if and only if all its quotient bundles have non-negative degree. As a result, the restriction of a generically semipositive sheaf or bundle to a sufficiently general curve is nef (say on a projective variety).

Theorem $\mathrm{C}$ is a variation on Miyaoka's semiposivity theorem [Miy87]. In the case where $X$ is smooth projective, it has been proved by Boucksom-Demailly-Păun-Peternell [BDPP13] that $X$ is uniruled if and only if $K_{X}$ is not pseudo-effective, so that we essentially recover the same statement. However, as opposed to Miyaoka's theorem, our result works in the context of singular log pairs and holds for (singular) Kähler spaces and not only for algebraic varieties - it was also proved in the smooth Kähler case by Cao [Cao13]. Also, as there exist rational (hence uniruled) surfaces with only klt singularities and for which $K_{X}$ is ample [Kol08, Example 43], our assumption that $K_{X}$ is pseudo-effective encompasses some new cases even when $D=0$.

Proof of Theorem $C$. Let us begin with the case $K_{X}+D$ pseudo-effective. Here again, we denote by $Y$ the singular original Kähler space, and choose a $\log$ resolution $\pi: X \rightarrow Y$ of $(Y, \Delta)$. We will show that for each coherent subsheaf $\mathscr{F} \subset T_{X}(-\log D)$ and every Kähler form $\omega_{0}$ on $Y$, we have $\int_{X} c_{1}(\mathscr{F}) \wedge\left(\pi^{*} \omega_{0}\right)^{n-1} \leqslant 0$. As before, this shows that each coherent subsheaf of $\mathscr{T}_{Y}(-\log \Delta)$ has non-positive slope with respect to $\omega$. We conclude by duality (consider a quotient $\mathcal{G}$ of $\Omega_{Y}^{1}(\log \Delta)$; it induces a subsheaf $\mathcal{G}^{*} \subset \mathscr{T}_{Y}(-\log \Delta)$ with non-positive slope, so that $\mathcal{G}$ has a non-negative slope).

We choose a log resolution $\pi: X \rightarrow Y$ of the pair $(Y, \Delta)$, so we have

$$
K_{X}+D+E=\pi^{*}\left(K_{Y}+\Delta\right),
$$

where $D=\sum D_{k}$ is a reduced snc divisor (containing the strict transform of $\Delta$ as well as an exceptional divisor with zero $\log$ discrepancy) and $E=\sum a_{i} E_{i}$ is an exceptional divisor with positive log discrepancies (that is, whose coefficients satisfy $a_{i}<1$, but are possibly negative).

We would like to endow $T_{X}(-\log D)$ with a singular metric having seminegative Ricci curvature outside $D \cup E$ in a weak sense; that is, we would like to solve

$$
\operatorname{Ric} \omega=-T+[D]+[E]
$$

for $\omega \in\left\{\pi^{*} \omega_{0}\right\}$ and $T \in \pi^{*}\left(K_{Y}+\Delta\right)$ a positive current. Unfortunately, there are obstructions to constructing such a metric (as this would amount to solving a Monge-Ampère equation with non-integrable right-hand side) and using it for our purposes (we would need to know its behavior near the singularities in order to perform integrations by parts, for example). Therefore, we will instead approximate a potential object of this type by regularizing the equation in the following fashion:

- Replace the cohomology class of $\omega$ by the Kähler class $\left\{\pi^{*} \omega_{0}+\delta \omega_{X}\right\}$ for some Kähler metric $\omega_{X}$.

- Replace $D$ by $(1-\delta) D$.

- Choose a positive current $T_{\delta} \in \pi^{*}\left(K_{Y}+\Delta\right)+\delta\left\{\omega_{X}\right\}$ with analytic singularities.

- Replace $[E]$ by a smooth $(1,1)$-form $\Theta_{\varepsilon}$. 


\section{H. Guenancia}

For the third item, we use Demailly's regularization theorem [Dem92] inside the big cohomology class $\pi^{*}\left(K_{Y}+\Delta\right)+\delta\left\{\omega_{X}\right\}$, while for the last item, we choose

$$
\Theta_{\varepsilon}=\sum_{i} a_{i}\left[\theta_{E_{i}}+d d^{c} \log \left(\left|s_{i}\right|^{2}+\varepsilon^{2}\right)\right]=\sum_{i} a_{i}\left(\frac{\varepsilon^{2}\left|D^{\prime} s_{i}\right|^{2}}{\left(\left|s_{i}\right|^{2}+\varepsilon^{2}\right)^{2}}+\frac{\varepsilon^{2} \theta_{E_{i}}}{|s|^{2}+\varepsilon^{2}}\right),
$$

where $s_{i}$ is a defining section for $E_{i}$ and $\theta_{E_{i}}$ is the Chern curvature of the smooth hermitian metric $|\cdot|_{i}$ chosen on $\mathcal{O}_{X}\left(E_{i}\right)$. Set $\theta_{E}:=\sum a_{i} \theta_{E_{i}}$. We also choose sections $\sigma_{k}$ of the components $D_{k}$ of $D$ and pick arbitrary smooth hermitian metrics $|\cdot|_{k}$ on $\mathcal{O}_{X}\left(D_{k}\right)$ whose curvature form will be denoted by $\theta_{D_{k}}$. Finally, set $\theta_{D}=\sum_{k} \theta_{D_{k}}$.

Let us now choose an arbitrary volume form $d V$ on $X$; one can find a (sup-normalized) potential $\psi_{\delta}$ for $T_{\delta}$ such that $T_{\delta}=-\operatorname{Ric}(d V)+\theta_{D}+\theta_{E}+\delta \omega_{X}+d d^{c} \psi_{\delta}$. In particular, there exists a uniform $C$ (independent of $\delta$ ) such that $d d^{c} \psi_{\delta} \geqslant-C \omega_{X}$. Moreover, as $T_{\delta}$ has analytic singularities, $\psi_{\delta}$ is smooth outside a proper analytic set $Z \subset X$ (one should observe that $Z$ depends on $\delta$, though); set $X_{0}:=X \backslash(D \cup Z)$. Thanks to Kołodziej's theorem [Koł98], one can find a bounded solution $\varphi=\varphi_{\delta, \varepsilon}$ of the following Monge-Ampère equation:

$$
\left(\pi^{*} \omega_{0}+\delta \omega_{X}+d d^{c} \varphi\right)^{n}=\frac{e^{\psi_{\delta}+C_{\delta, \varepsilon}} d V}{\prod_{i}\left(\left|s_{i}\right|^{2}+\varepsilon^{2}\right)^{a_{i}} \prod_{k}\left|\sigma_{k}\right|^{2(1-\delta)}},
$$

where $C_{\delta, \varepsilon}$ is a normalizing constant which is bounded uniformly with respect to $\varepsilon$ (but not $\delta$ !). Although the solution $\omega_{\delta, \varepsilon}:=\pi^{*} \omega_{0}+\delta \omega_{X}+d d^{c} \varphi$ strongly depends on the parameters $\delta$ and $\varepsilon$, we choose not to mention this dependence anymore to lighten the notation. So in the following, $\omega=\pi^{*} \omega_{0}+\delta \omega_{X}+d d^{c} \varphi$ will denote the solution of equation (5.3). It is straightforward to check that

$$
\operatorname{Ric} \omega=-T_{\delta}+(1-\delta)[D]+\delta \theta_{D}+\Theta_{\varepsilon}+\delta \omega_{X}
$$

in the sense of currents; this is the regularized version of (5.1).

It is crucial to understand the singularities of $\omega$. Let us first fix $\delta$ and $\varepsilon$. We would like to use the Laplacian estimate [GP16, Proposition 2.1], so we need to properly regularize $e^{\psi_{\delta}} / \prod\left|\sigma_{k}\right|^{2(1-\delta)}$. On the one hand, the Demailly regularization theorem enables us to regularize $\psi_{\delta}$ by smooth sup-normalized functions $\psi_{\delta, \eta}$ such that $d d^{c} \psi_{\delta, \eta} \geqslant-C \omega_{X}$ (up to increasing $C$ ) and on the other hand, one can replace $1 / \prod\left|\sigma_{k}\right|^{2(1-\delta)}$ by $1 / \prod\left(\left|\sigma_{k}\right|^{2}+\eta^{2}\right)^{1-\delta}$. Everything is in order to apply [GP16, Proposition 2.1], so we obtain that $\omega$ is dominated by a metric with conic singularities with cone angle $2 \pi \delta$ along $D$; that is, there exists a $C_{\delta, \varepsilon}$ such that

$$
\omega \leqslant C\left(\omega_{X}+\sum_{k} \frac{\left\langle D^{\prime} \sigma_{k}, D^{\prime} \sigma_{k}\right\rangle}{\left|\sigma_{k}\right|^{2(1-\delta)}}\right)
$$

so, in particular, $\omega$ is smooth outside $D \cup Z$ (by standard bootstrapping arguments). As a result, we can find a family of cut-off functions $\left(\chi_{\eta}\right)_{\eta>0}$ vanishing near $D \cup Z$ and converging to the indicatrix of the complement of this set, such that $\left\|\operatorname{tr}_{\omega}\left(d d^{c} \chi_{\eta}\right)\right\|_{L^{1}\left(\omega^{n}\right)} \rightarrow 0$. This family can be made explicit if $Z$ is empty; cf. [CGP13, Section 9]. Else, one considers a log resolution of the ideal corresponding to $D \cup Z$ (with its reduced structure) and constructs the family upstairs similarly thanks to $(5.5)$.

Finally, applying Proposition 2.1 to the Monge-Ampère equation (5.3) yields the convergence

$$
\int_{X_{0}} \frac{\varepsilon^{2}}{|s|^{2}+\varepsilon^{2}} \omega_{X} \wedge \omega^{n-1} \rightarrow 0
$$

when $\varepsilon$ tends to zero ( $\delta>0$ being fixed) thanks to the same arguments as in Lemma 3.7, 
whether $(s=0)$ cuts out a component of $E$ or one of $D$. To be rigorous, one should only apply Proposition 2.1 once (5.3) is regularized. Basically, one needs to replace $\left|\sigma_{k}\right|^{-2(1-\delta)}$ by $\left(\left|\sigma_{k}\right|^{2}+\eta^{2}\right)^{\delta-1}$ for $\eta>0$ and $\psi_{\delta}$ by a uniformly quasi-psh smooth approximant, which is possible thanks to [Dem92].

Back to our problem, we start with a generically injective morphism $\mathscr{F} \rightarrow T_{X}(-\log D)$. If $r=\operatorname{rk}(\mathscr{F})$, then this morphism induces $L=\left(\Lambda^{r} \mathscr{F}\right)^{* *} \rightarrow \Lambda^{r} T_{X}(-\log D)$. As $X$ is smooth and $L$ is reflexive of rank 1, it is actually locally free, so the above morphism yields a non-zero section $u$ of the bundle $\Lambda^{r} T_{X}(-\log D) \otimes L^{-1}$. We want to endow this bundle with a metric. For $L$, we just choose an arbitrary smooth hermitian metric $h_{L}$, and for $\Lambda^{r} T_{X}(-\log D)$, we would like to use $\omega$. As $\omega$ is singular along $D \cup Z$, we need to work on $X_{0}=X \backslash(D \cup Z)$, where $\omega$ is a smooth Kähler metric and induces a genuine hermitian metric on $T_{X}(-\log D)_{\mid X_{0}} \simeq T_{X \mid X_{0}}$, and hence on $\Lambda^{r} T_{X \mid X_{0}}$. Let us call this metric $h$; we can then measure $u$ with respect to $h \otimes h_{L}^{-1}$. For any number $\lambda>0$, we have on $X_{0}$

$$
\begin{aligned}
d d^{c} \log \left(|u|^{2}+\lambda^{2}\right)= & \frac{1}{|u|^{2}+\lambda^{2}}\left(\left|D^{\prime} u\right|^{2}-\frac{\left|\left\langle D^{\prime} u, u\right\rangle\right|^{2}}{|u|^{2}+\lambda^{2}}\right. \\
& \left.-\left\langle\Theta\left(\Lambda^{r} T_{X}(-\log D) \otimes L^{-1}, h \otimes h_{L}^{-1}\right) u, u\right\rangle\right) .
\end{aligned}
$$

Here, for a (vector-valued) (1,0)-form $\alpha$, we used the notation $|\alpha|^{2}:=\alpha \wedge \bar{\alpha}$. As $\left|\left\langle D^{\prime} u, u\right\rangle\right|^{2} \leqslant$ $\left|D^{\prime} u\right|^{2} \cdot|u|^{2}$, we deduce from the equality above

$$
d d^{c} \log \left(|u|^{2}+\lambda^{2}\right) \geqslant \frac{|u|^{2}}{|u|^{2}+\lambda^{2}}\left(\Theta\left(L, h_{L}\right)-\frac{\left\langle\Theta\left(\Lambda^{r} T_{X}(-\log D), h\right) u, u\right\rangle}{|u|^{2}}\right),
$$

where the action of $\Theta\left(\Lambda^{r} T_{X}(-\log D), h\right)$ is extended to $\Lambda^{r} T_{X}(-\log D) \otimes L^{-1}$ by tensoring it with $\operatorname{Id}_{L^{-1}}$. Let us observe that $u$ is a section of $\Lambda^{r} T_{X}(-\log D) \otimes L^{-1}$ and that $\omega$ is dominated by a conic metric along $D$; cf. (5.5). Therefore $|u|$ is globally bounded on $X_{0}$ (of course, the bound depends on both $\delta$ and $\varepsilon$ ).

Now, we wedge inequality (5.8) with $\omega^{n-1}$ and try to integrate it on $X_{0}$. In order to legitimate this, we first need to multiply by the cut-off function $\chi_{\eta}$ mentioned a few lines above. Using the Stokes theorem, we get

$$
-\int_{X} \log \left(|u|^{2}+\lambda^{2}\right) d d^{c} \chi_{\eta} \wedge \omega^{n-1} \geqslant \int_{X} \frac{\chi_{\eta}|u|^{2}}{|u|^{2}+\lambda^{2}}\left(\Theta\left(L, h_{L}\right)-\frac{\left\langle\Theta\left(\Lambda^{r} T_{X}, h\right) u, u\right\rangle}{|u|^{2}}\right) \wedge \omega^{n-1} .
$$

Our goal is to study the convergence of each of the terms appearing in this inequality when the parameters $\eta, \lambda, \varepsilon, \delta$ (in this order) converge to zero.

Let us begin with the term on the left-hand side. As $\log \left(|u|^{2}+\lambda^{2}\right)$ is globally bounded on $X_{0}$ (since $\lambda>0$ ) and $\operatorname{tr}_{\omega}\left(d d^{c} \chi_{\eta}\right) \rightarrow 0$ in $L^{1}\left(X_{0}, \omega^{n}\right)$, this term converges to zero when $\eta \rightarrow 0$, the other parameters $(\lambda, \varepsilon, \delta)$ being fixed.

As $\omega$ has bounded potentials, the dominated convergence theorem guarantees that the integral

$$
\int_{X} \frac{\chi_{\eta}|u|^{2}}{|u|^{2}+\lambda^{2}} \Theta\left(L, h_{L}\right) \wedge \omega^{n-1}
$$

converges to $c_{1}(L) \cdot\{\omega\}^{n-1}=c_{1}(L) \cdot\left(\pi^{*}\left(K_{Y}+\Delta\right)+\delta\left\{\omega_{X}\right\}\right)^{n-1}$ when $\eta$ and $\lambda$ go to zero. This is a standard argument that we will use repeatedly, so here is a justification. If $T$ is a positive current and $\varphi$ a bounded quasi-psh function, then $d d^{c} \varphi \wedge T:=d d^{c}(\varphi T)$ is a (signed) measure with zero integral on $X$ (just evaluate this current against the constant function 1 ). 


\section{H. Guenancia}

Next, we apply this to $T=\Theta\left(L, h_{L}\right) \wedge \omega^{n-1}$ to get $\int_{X} \Theta\left(L, h_{L}\right) \wedge \omega^{n-1}=\int_{X} \Theta\left(L, h_{L}\right) \wedge \omega^{n-1} \wedge$ $\left(\pi^{*} \omega_{0}+\delta \omega_{X}\right)$, and inductively, we find $\int_{X} \Theta\left(L, h_{L}\right) \wedge\left(\pi^{*} \omega_{0}+\delta \omega_{X}\right)^{n-1}=c_{1}(L) \cdot\left(\pi^{*}\left(K_{Y}+\Delta\right)+\right.$ $\left.\delta\left\{\omega_{X}\right\}\right)^{n-1}$.

The remaining term is the more subtle to deal with. First, let us introduce notation: Let $V$ be a complex vector space of dimension $n$, let $1 \leqslant r \leqslant n$ be an integer, and let $f \in \operatorname{End}(V)$. We denote by $f^{\wedge r}$ the endomorphism of $\Lambda^{r} V$ defined by

$$
f^{\wedge r}\left(v_{1} \wedge \cdots \wedge v_{r}\right):=\sum_{i=1}^{r} v_{1} \wedge \cdots v_{i-1} \wedge f\left(v_{i}\right) \wedge v_{i+1} \wedge \cdots \wedge v_{r} .
$$

Let us add that if $V$ has an hermitian structure and if $f$ is hermitian semipositive, then so is $f^{\wedge} r$ with the induced metric, and we have $\operatorname{tr}\left(f^{\wedge r}\right) \leqslant\left(\begin{array}{l}n \\ r\end{array}\right) \operatorname{tr}(f)$. Now, we can easily check the following identity:

$$
n \Theta\left(\Lambda^{r} T_{X}, h\right) \wedge \omega^{n-1}=(\sharp \operatorname{Ric} \omega)^{\wedge r} \omega^{n} .
$$

But on $X_{0}$, we have from (5.4) that Ric $\omega=-T_{\delta}+\delta \theta_{D}+\Theta_{\varepsilon}+\delta \omega_{X}$, hence Ric $\omega \leqslant \delta \theta_{D}+\Theta_{\varepsilon}+\delta \omega_{X}$, so there are now two terms to be controlled.

The first one involves $\delta \omega_{X}$ (we can treat $\delta \theta_{D}$ in the same way):

$$
0 \leqslant\left\langle\sharp\left(\omega_{X}\right)^{\wedge r} u, u\right\rangle \omega^{n} \leqslant\left(\begin{array}{c}
n \\
r
\end{array}\right) \operatorname{tr}\left(\sharp \omega_{X}\right)|u|^{2} \omega^{n}=\left(\begin{array}{c}
n \\
r
\end{array}\right) \operatorname{tr}_{\omega}\left(\omega_{X}\right)|u|^{2} \omega^{n}=n\left(\begin{array}{l}
n \\
r
\end{array}\right)|u|^{2} \omega_{X} \wedge \omega^{n-1} .
$$

Hence, we get

$$
0 \leqslant \int_{X} \frac{\chi_{\eta}|u|^{2}}{|u|^{2}+\lambda^{2}} \cdot \frac{\left\langle\left(\sharp\left(\delta \omega_{X}\right)\right)^{\wedge r} u, u\right\rangle \omega^{n}}{|u|^{2}} \leqslant n\left(\begin{array}{c}
n \\
r
\end{array}\right) \delta \int_{X_{0}} \omega_{X} \wedge \omega^{n-1}
$$

and the right-hand side equals $n\left(\begin{array}{l}n \\ r\end{array}\right) \delta\left\{\omega_{X}\right\} \cdot\{\omega\}^{n-1}$ (because $\omega$ has bounded potentials), which is a cohomological quantity depending only on $\delta$ and converging to zero when this parameter does so.

So now we need to control $\left\langle\left(\sharp \Theta_{\varepsilon}\right)^{\wedge r} u, u\right\rangle \omega^{n}$. We would like to view this term as a cohomological one, but the $\sharp$ operation makes it difficult. So we split this term into a main positive (or negative according to the sign of $a_{i}$ ) part and a smaller correction factor; one can apply basic inequalities to the term with a sign to recover in the end cohomological terms up to a small error factor.

Recall (5.2),

$$
\Theta_{\varepsilon}=\sum a_{i}\left(\frac{\varepsilon^{2}\left|D^{\prime} s_{i}\right|^{2}}{\left(\left|s_{i}\right|^{2}+\varepsilon^{2}\right)^{2}}+\frac{\varepsilon^{2} \theta_{E_{i}}}{|s|^{2}+\varepsilon^{2}}\right)
$$

where $\theta_{E_{i}}$ is the curvature form of the hermitian metric chosen on $\mathcal{O}_{X}\left(E_{i}\right)$. By linearity, it is enough to consider each of these summands separately. So we pick one and drop the index $i$ to simplify the notation. So we have $\Theta_{\varepsilon}=a(\beta+\gamma)$, where

$$
\beta=\frac{\varepsilon^{2}\left|D^{\prime} s\right|^{2}}{\left(|s|^{2}+\varepsilon^{2}\right)^{2}} \quad \text { and } \quad \gamma=\frac{\varepsilon^{2} \theta}{|s|^{2}+\varepsilon^{2}} ;
$$

remember that these forms are smooth as long as $\varepsilon>0$. Let us start with $\gamma$. There exists a constant $C>0$ such that $\pm \gamma \leqslant C \varepsilon^{2} /\left(|s|^{2}+\varepsilon^{2}\right) \omega_{X}$. As the $\sharp$ and ${ }^{\wedge} r$ operations preserve positivity, we get $\pm(\sharp \gamma)^{\wedge r} \omega^{n} \leqslant C \varepsilon^{2} /\left(|s|^{2}+\varepsilon^{2}\right)\left(\sharp \omega_{X}\right)^{\wedge r} \omega^{n}$. But $\sharp \omega_{X}$ is a positive endomorphism whose trace is $\operatorname{tr}_{\omega} \omega_{X}$, and therefore $\left(\sharp \omega_{X}\right)^{\wedge r} \leqslant\left(\begin{array}{l}n \\ r\end{array}\right) \operatorname{tr}_{\omega}\left(\omega_{X}\right)$ Id. Consequently,

$$
\frac{ \pm\left\langle(\sharp \gamma)^{\wedge r} u, u\right\rangle \omega^{n}}{|u|^{2}} \leqslant \frac{C \varepsilon^{2}}{|s|^{2}+\varepsilon^{2}} \omega_{X} \wedge \omega^{n-1}
$$

for some $C>0$ independent of $\varepsilon$. From (5.6) and the dominated convergence theorem, one 
deduces that the integral

$$
\int_{X} \frac{\chi_{\eta}|u|^{2}}{|u|^{2}+\lambda^{2}} \cdot \frac{\left\langle(\sharp \gamma)^{\wedge r} u, u\right\rangle \omega^{n}}{|u|^{2}}
$$

converges to zero when $\eta, \lambda$ and $\varepsilon$ go to zero.

The last term to control is the one involving $\beta$. We know that $\beta$ is non-negative, so $(\sharp \beta)^{\wedge r} \omega^{n} \leqslant$ $n\left(\begin{array}{l}n \\ r\end{array}\right) \beta \wedge \omega^{n-1} \mathrm{Id}$, so

$$
\begin{aligned}
0 \leqslant \int_{X} \frac{\chi_{\eta}|u|^{2}}{|u|^{2}+\lambda^{2}} \cdot \frac{\left\langle(\sharp \beta)^{\wedge r} u, u\right\rangle \omega^{n}}{n|u|^{2}} & \leqslant C \int_{X} \frac{\chi_{\eta}|u|^{2}}{|u|^{2}+\lambda^{2}} \cdot \beta \wedge \omega^{n-1} \\
& \leqslant C \int_{X_{0}} \beta \wedge \omega^{n-1} \\
& =C\left(\int_{X_{0}}(\beta+\gamma) \wedge \omega^{n-1}-\int_{X_{0}} \gamma \wedge \omega^{n-1}\right) \\
& =C\left(c_{1}\left(E_{i}\right) \cdot\{\omega\}^{n-1}-\int_{X_{0}} \gamma \wedge \omega^{n-1}\right),
\end{aligned}
$$

where we have used the fact that $\omega$ has bounded potentials (so that none of its powers charge a pluripolar set). We have already observed that the second integral converges to zero when $\varepsilon \rightarrow 0$. As for the first term, it is cohomological (independent of $\varepsilon$ ) and it equals $\delta^{n-1} c_{1}\left(E_{i}\right) \cdot\left\{\omega_{X}\right\}^{n-1}$ as $E_{i}$ is $\pi$-exceptional; thus it converges to zero when $\delta$ goes to zero.

Finally, we have shown that if we first make $\eta$ go to zero, and then $\lambda, \varepsilon$ and at last $\delta$, inequality (5.9) becomes $L \cdot\left\{\pi^{*} \omega_{0}\right\}^{n-1} \leqslant 0$, which is equivalent to $c_{1}(\mathscr{F}) \cdot\left\{\pi^{*} \omega_{0}\right\}^{n-1} \leqslant 0$ or, using (3.1), to $c_{1}(\mathscr{G}) \cdot\left\{\pi^{*} \omega_{0}\right\}^{n-1} \leqslant 0$, which is the expected generic semipositivity inequality for the sheaf of logarithmic forms $\Omega_{Y}^{1}(\log \Delta)$.

The case where $-\left(K_{Y}+\Delta\right)$ is pseudo-effective is very similar. Again, it is enough to show that every coherent subsheaf $\mathscr{F} \subset \Omega_{X}^{1}(\log D)$ has non-positive slope with respect to any "polarization" $\pi^{*} \omega_{0}^{n-1}$ pulled back from $Y$. We solve the same Monge-Ampère equation, but now the metric satisfies Ric $\omega_{\varepsilon, \delta}=T_{\delta}+(1-\delta)[D]+\delta \theta_{D}+\Theta_{\varepsilon}-\delta \omega_{X}$, where $\omega_{\varepsilon, \delta} \in \pi^{*} \omega_{0}+\delta \omega_{X}$ and $T_{\delta}$ is a positive current in $c_{1}\left(-\pi^{*}\left(K_{Y}+\Delta\right)\right)+\delta\left\{\omega_{X}\right\}$, so that $\operatorname{Ric} \omega_{\varepsilon, \delta} \geqslant \delta \theta_{D}+\Theta_{\varepsilon}-\delta \omega_{X}$. Now, $\Theta\left(\Omega_{X}^{1}\right)=-\Theta\left(T_{X}\right)^{*}$, so that $\operatorname{tr}_{\omega} \Theta\left(\Omega_{X}^{1}\right)=-\sharp \operatorname{Ric} \omega$. We can now run the same computations as above and get the non-positivity of the slope of $\mathscr{F}$.

We can now move on to the proof of the polystability of the tangent sheaf of singular CalabiYau varieties.

Proof of the result below Theorem C. Let us start with a "polarization" $\omega_{0}$ on $Y$, and take the notation of the proof above, where we work on a resolution $\pi: X \rightarrow Y$ of the Calabi-Yau variety $Y$ and denote the exceptional divisor of $\pi$ by $E$. As the slope of $\mathscr{T}_{Y}$ vanishes, Theorem $\mathrm{C}$ already gives the $\omega$-semistability of $\mathscr{T}_{Y}$. Now, suppose that there exists a reflexive subsheaf $\mathscr{F} \subset \mathscr{T}_{Y}$ whose slope with respect to $\omega_{Y}$ vanishes too. As in step 4 of the proof of Theorem 3.5 (the case $K_{Y}$ ample), one chooses $\mathscr{G}$ of minimal rank, and it suffices to find a holomorphic complement of $\mathscr{G}$ inside $\mathscr{T}_{Y}$. Therefore we are reduced to showing that one can complement over $X \backslash E$ any reflexive subsheaf $\mathscr{F} \subset \mathscr{T}_{X}$ whose slope with respect to $\pi^{*} \omega$ vanishes. We denote by $W$ the minimal analytic set (of codimension at least 2 as $\mathscr{F}$ is reflexive) outside which $\mathscr{F}$ is a subbundle of $T_{X}$.

We run the proof of Theorem $\mathrm{C}$ above, and as $K_{Y}$ is numerically trivial, one can choose $T_{\delta}=0$ from the very beginning (and $D=0$ of course, as well as $\lambda=1$ ). So we end up with a family of 


\section{H. Guenancia}

Kähler metrics $\omega:=\omega_{\varepsilon, \delta}$ cohomologous to $\pi^{*} \omega_{0}+\delta \omega_{X}$ and solving Ric $\omega=\Theta_{\varepsilon}$, where $\Theta_{\varepsilon}$ (see $(5.2))$ is a smooth approximant of the current of integration along $E$, with $E$ the exceptional divisor representing $-K_{X}$.

We know (for example, from [EGZ09]) that $\omega_{\varepsilon, \delta}$ converges in $\mathscr{C}_{\text {loc }}^{\infty}(X \backslash E)$ to a smooth Ricci-flat metric $\omega_{\infty}$ inducing a smooth hermitian metric $h_{\infty}$ on $T_{X_{\mid X \backslash E}}$.

Inequality (5.8) must become an equality at the limit, so the term we neglected to go from (5.7) to $(5.8)$ necessarily converges to zero. This term is (remember that $\lambda=1$ )

$$
\frac{1}{|u|^{2}+1}\left(\left|D^{\prime} u\right|^{2}-\frac{\left|\left\langle D^{\prime} u, u\right\rangle\right|^{2}}{|u|^{2}+1}\right) \geqslant \frac{\left|D^{\prime} u\right|^{2}}{\left(|u|^{2}+1\right)^{2}}
$$

thanks to the Cauchy-Schwarz inequality.

Fatou's lemma shows that $\left|D^{\prime} u\right|_{\omega_{\infty}}=0$ on $X \backslash(W \cup E)$, and therefore $\operatorname{det} \mathscr{F}$ is a parallel subbundle of $T_{X}$ on this locus. By simple algebraic manipulations, this implies that $\mathscr{F}$ itself is a parallel subbundle of $T_{X}$. Therefore we get a holomorphic splitting $\mathscr{T}_{X}=\mathscr{F} \oplus \mathscr{F} \perp$ over $X \backslash(W \cup E)$. We can push forward this identity to $Y$, which by reflexivity gives us the expected complement of $\mathscr{G}$ inside $\mathscr{T}_{Y}$, as $\pi(E \cup W)$ has codimension at least 2 .

\section{A Bogomolov-type inequality for Chern numbers}

If $X$ is a singular variety (endowed with a Kähler-Einstein metric), then it is in general not possible to reproduce the arguments of Yau and work directly with the metric on the smooth locus to get an appropriate inequality between Chern numbers - this would require us to somehow control the curvature of the metric near the singularities, but this seems out of reach for now. Instead, we will rely on the so-called Bogomolov-Gieseker inequality, which asserts that any reflexive sheaf $E$ of rank $r$ on a smooth projective surface which is semistable with respect to some given polarization $H$ satisfies

$$
c_{1}(E)^{2} \leqslant \frac{2 r}{r-1} c_{2}(E)
$$

The proof of the Bogomolov inequality, generalized by Miyaoka in higher dimension [Miy87, Corollary 4.7], consists in analyzing the high powers of the semistable bundle $\mathcal{O}_{\mathbb{P}(E)}(1) \otimes \frac{1}{r} \operatorname{det} E$. This bundle has degree zero, hence its twist by an anti-effective divisor cannot admit any sections. Identifying the leading-order term in the Riemann-Roch formula leads to inequality (6.1); we refer to [MP97, Section 3] for more details.

The argument uses the Mehta-Ramanathan theorem to reduce the general case to the case of surfaces, so it is important to work with an ample polarization. However, [Sug90, Theorem 6.1] showed that we can get a slightly weaker statement for singular varieties, by proving that if $X$ is $\mathbb{Q}$-Gorenstein and $\pi: Y \rightarrow X$ is a resolution of singularities, then any rank $r$ bundle $E$ on $Y$ which is semistable with respect to $\pi^{*} H$ for some ample divisor $H$ on $X$ satisfies the following Bogomolov inequality:

$$
c_{1}(E)^{2} \cdot\left(\pi^{*} H\right)^{n-2} \leqslant \frac{2 r}{r-1} c_{2}(E) \cdot\left(\pi^{*} H\right)^{n-2} .
$$

Combining this with Enoki's result about the semistability of the tangent sheaf for canonical varieties with ample (respectively, trivial) canonical class, Sugiyama obtained the inequality above with $H=K_{X}$ (respectively, $H$ arbitrary). Actually, using the base-point-free theorem, he could also generalize it to the case where $K_{X}$ is nef and big, even essentially assuming only that 
$K_{X}$ is nef (relying on Miyaoka's generic semipositivity theorem for $c_{2}$ ).

Sugiyama's arguments can be transposed to the case of a log pair $(X, D)$ such that $K_{X}+D$ is $\mathbb{Q}$-Cartier without any significant changes. So we can use these ideas in our setting; combined with Theorem A, they yield the following result. The context is as follows: let $(X, D)$ be a projective $\log$ canonical pair of dimension $n$ and $\pi: Y \rightarrow X$ a log resolution. We denote by $\Delta$ the sum of the strict transform of $D$ and the exceptional divisor with zero log discrepancy; to put it in a different way, $K_{Y}+\Delta-\pi^{*}\left(K_{X}+D\right)$ is an exceptional divisor with coefficients strictly greater than -1 . If $K_{X}+D$ is ample (respectively, numerically trivial), then the proof of Theorem A shows that $\mathscr{T}_{Y}(-\log \Delta)$ is semistable with respect to $\pi^{*}\left(K_{X}+D\right)$ (respectively, $\pi^{*} H$ for any ample line bundle $H$ on $X$ ).

Putting together the previous observations, we get Theorem 1.1, stated in the introduction.

\section{ACKNOWLEDGEMENTS}

This work was initiated near the end of my Ph.D. thesis, and I am grateful to my advisors Sébastien Boucksom and Mihai Păun, who generously shared their ideas and helped me develop this article. I am particularly indebted to Mihai Păun, who shared his unpublished work [CP14] with me and followed the development of this paper with interest. Finally, I would like to thank Patrick Graf for helpful discussions, and the referee for reading the manuscript extremely carefully and pointing out several inaccuracies.

\section{REFERENCES}

Ale96 V. Alexeev, Log canonical singularities and complete moduli of stable pairs, 1996, arXiv:alggeom/9608013.

Aub78 T. Aubin, Équations du type Monge-Ampère sur les variétés kählériennes compactes, Bull. Sci. Math. 102 (1978), no. 1, 63-95.

BBEGZ11 R. J. Berman, S. Boucksom, P. Eyssidieux, V. Guedj and Z. Zeriahi, Kähler-Einstein metrics and the Kähler-Ricci flow on log-Fano varieties, 2011, arXiv:1111.7158.

BBGZ13 R. J. Berman, S. Boucksom, V. Guedj and A. Zeriahi, A variational approach to complex Monge-Ampère equations, Publ. Math. Inst. Hautes Études Sci. 117 (2013), 179-245; http: //dx.doi.org/10.1007/s10240-012-0046-6.

BDPP13 S. Boucksom, J.-P. Demailly, M. Păun and T. Peternell, The pseudo-effective cone of a compact Kähler manifold and varieties of negative Kodaira dimension, J. Algebraic Geom. 22 (2013), no. 2, 201-248; http://dx.doi.org/10.1090/S1056-3911-2012-00574-8.

BG14 R. J. Berman and H. Guenancia, Kähler-Einstein metrics on stable varieties and log canonical pairs, Geom. Funct. Anal. 24 (2014), no. 6, 1683-1730; http://dx.doi.org/10.1007/ s00039-014-0301-8.

BHPS13 B. Bhatt, W. Ho, Z. Patakfalvi and C. Schnell, Moduli of products of stable varieties, Compos. Math. 149 (2013), no. 12, 2036-2070; http://dx.doi.org/10.1112/S0010437X13007288.

Bło12 Z. Błocki, The Calabi-Yau theorem, Complex Monge-Ampère Equations and Geodesics in the Space of Kähler Metrics, Lecture Notes in Math., vol. 2038 (Springer, Heidelberg, 2012), 201-227; http://dx.doi.org/10.1007/978-3-642-23669-3_5.

Cao13 J. Cao, A remark on compact Kähler manifolds with nef anticanonical bundles and its applications, 2013, arXiv:1305.4397.

CGP13 F. Campana, H. Guenancia and M. Păun, Metrics with cone singularities along normal crossing divisors and holomorphic tensor fields, Ann. Sci. Éc. Norm. Supér. (4) 46 (2013), no. 6, 879-916. 


\section{H. Guenancia}

Che68 S.-S. Chern, On holomorphic mappings of hermitian manifolds of the same dimension, Entire Functions and Related Parts of Analysis (Proc. Sympos. Pure Math., La Jolla, Calif., 1966) (Amer. Math. Soc., Providence, R.I., 1968), 157-170.

CP13 F. Campana and M. Păun, Orbifold generic semi-positivity: an application to families of canonically polarized manifolds, 2013, arXiv:1303.3169.

CP14 Positivity properties of the bundle of logarithmic tensors on compact Kähler manifolds, 2014, arXiv:1407.3431.

Dem92 J.-P. Demailly, Regularization of closed positive currents and intersection theory, J. Algebraic Geom. 1 (1992), no. 3, 361-409.

DM69 P. Deligne and D. Mumford, The irreducibility of the space of curves of given genus, Publ. Math. Inst. Hautes Études Sci. 36 (1969), 75-109; http://dx.doi.org/10.1007/ BF02684599.

Don83 S. K. Donaldson, A new proof of a theorem of Narasimhan and Seshadri, J. Differential Geom. 18 (1983), no. 2, 269-277; http://projecteuclid.org/euclid.jdg/1214437664.

Don85_ Anti self-dual Yang-Mills connections over complex algebraic surfaces and stable vector bundles, Proc. London Math. Soc. 50 (1985), no. 1, 1-26; http://dx.doi.org/10. 1112/plms/s3-50.1.1.

Don87 _ Infinite determinants, stable bundles and curvature, Duke Math. J. 54 (1987), no. 1, 231-247; http://dx.doi.org/10.1215/S0012-7094-87-05414-7.

EGZ09 P. Eyssidieux, V. Guedj and A. Zeriahi, Singular Kähler-Einstein metrics, J. Amer. Math. Soc. 22 (2009), no. 3, 607-639; http://dx.doi.org/10.1090/S0894-0347-09-00629-8.

Eno88 I. Enoki, Stability and negativity for tangent sheaves of minimal Kähler spaces, Geometry and Analysis on Manifolds (Katata/Kyoto, 1987), Lecture Notes in Math., vol. 1339 (Springer, Berlin, 1988), 118-126; http://dx.doi.org/10.1007/BFb0083051.

GKP11 D. Greb, S. Kebekus, and T. Peternell, Singular spaces with trivial canonical class, Minimal Models and Extremal Rays, Proceedings of the Conference in Honor of Shigefumi Mori's 60th Birthday, Adv. Stud. Pure Math. (Kinokuniya Publishing House, Tokyo), to appear; arXiv:1110.5250.

GP16 H. Guenancia and M. Păun, Conic singularities metrics with prescribed Ricci curvature: the case of general cone angles along normal crossing divisors, J. Differential Geom. 103 (2016), no. 1, 15-57; http://projecteuclid.org/euclid.jdg/1460463562.

Gue14 H. Guenancia, Kähler-Einstein metrics with mixed Poincaré and cone singularities along a normal crossing divisor, Ann. Inst. Fourier (Grenoble) 64 (2014), no. 3, 1291-1330; http: //dx.doi.org/10.5802/aif.2881.

GW16 H. Guenancia and D. Wu, On the boundary behavior of Kähler-Einstein metrics on log canonical pairs, Math. Ann., published online on 17 October 2015, http://dx.doi.org/10.1007/ s00208-015-1306-9, to appear in print.

GZ07 V. Guedj and A. Zeriahi, The weighted Monge-Ampère energy of quasiplurisubharmonic functions, J. Funct. Anal. 250 (2007), no. 2, 442-482; http://dx.doi.org/10.1016/j.jfa. 2007. 04.018.

Har71 R. Hartshorne, Ample vector bundles on curves, Nagoya Math. J. 43 (1971), 73-89; http: //projecteuclid.org/euclid.nmj/1118798366.

Har77 _ Algebraic geometry, Grad. Texts in Math. vol. 52 (Springer-Verlag, New York Heidelberg, 1977); http://dx.doi.org/10.1007/978-1-4757-3849-0.

Har80 Stable reflexive sheaves, Math. Ann. 254 (1980), no. 2, 121-176; http://dx.doi. org/10.1007/BF01467074.

HL10 D. Huybrechts and M. Lehn, The geometry of moduli spaces of sheaves, second ed., Cambridge Math. Lib. (Cambridge Univ. Press, Cambridge, 2010); http://dx . doi .org/10.1017/ CB09780511711985. 


\section{SEMistability OF THE TANGENT SHEAF OF SINGULAR VARIETIES}

JMR16 T. Jeffres, R. Mazzeo and Y. Rubinstein, Kähler-Einstein metrics with edge singularities, Ann. of Math. 183 (2016), no. 1, 95-176; http://dx.doi.org/10.4007/annals.2016.183.1.3.

KM98 J. Kollár and S. Mori, Birational geometry of algebraic varieties, Cambridge Tracts in Math., vol.134 (Cambridge Univ. Press, Cambridge, 1998); http://dx.doi.org/10.1017/ CB09780511662560.

KobR84 R. Kobayashi, Kähler-Einstein metric on an open algebraic manifold, Osaka J. Math. 21 (1984), no. 2, 399-418; http://projecteuclid.org/euclid.ojm/1200777118.

KobS82 S. Kobayashi, Curvature and stability of vector bundles, Proc. Japan Acad. Ser. A Math. Sci. 58 (1982), no. 4, 158-162; http://dx.doi.org/10.3792/pjaa.58.158.

KobS87 _ Differential geometry of complex vector bundles, Publ. Math. Soc. Japan, vol. 15 (Princeton Univ. Press, Princeton, NJ; Iwanami Shoten, Tokyo, 1987); http://dx.doi.org/ 10.1515/9781400858682.

Kol96 J. Kollár, Rational curves on algebraic varieties, Ergeb. Math. Grenzgeb. (3), vol. 32 (Springer-Verlag, Berlin, 1996); http://dx.doi.org/10.1007/978-3-662-03276-3.

Kol08 J. Kollár, Is there a topological Bogomolov-Miyaoka-Yau inequality?, Pure Appl. Math. Q. 4 (2008), no. 2, 203-236; http://dx.doi.org/10.4310/PAMQ.2008.v4.n2.a1.

Kol98 S. Kołodziej, The complex Monge-Ampère equation, Acta Math. 180 (1998), no. 1, 69-117; http://dx.doi.org/10.1007/BF02392879.

Kov13 S. J. Kovács, Singularities of stable varieties, Handbook of Moduli. Vol. II, Adv. Lect. Math., vol. 25 (Int. Press, Somerville, MA, 2013); 159-203.

KSB88 J. Kollár and N.I. Shepherd-Barron, Threefolds and deformations of surface singularities, Invent. Math. 91 (1988), no. 2, 299-338; http://dx.doi.org/10.1007/BF01389370.

Laz04 R. Lazarsfeld, Positivity in algebraic geometry. I. Classical setting: line bundles and linear series, Ergeb. Math. Grenzgeb. (3), vol. 48 (Springer-Verlag, Berlin, 2004).

Lu68 Y.-C. Lu, Holomorphic mappings of complex manifolds, J. Differential Geom. 2 (1968), 299312; http://projecteuclid.org/euclid.jdg/1214428442.

Lüb83 M. Lübke, Stability of Einstein-Hermitian vector bundles, Manuscripta Math. 42 (1983), no. 2, 245-257; http://dx.doi.org/10.1007/BF01169586.

Miy83 Y. Miyaoka, On the affine-ruledness of algebraic varieties, Algebraic Geometry (Tokyo/Kyoto, 1982), Lecture Notes in Math., vol. 1016 (Springer, Berlin, 1983), 449-489; http://dx.doi. org/10.1007/BFb0099975,

Miy87 Y. Miyaoka, The Chern classes and Kodaira dimension of a minimal variety, Algebraic Geometry (Sendai, 1985), Adv. Stud. Pure Math., vol. 10 (North-Holland, Amsterdam, 1987), 449-476.

MP97 Y. Miyaoka and T. Peternell, Geometry of higher-dimensional algebraic varieties, DMV Seminar, vol. 26 (Birkhäuser Verlag, Basel, 1997); http://dx.doi.org/10.1007/ 978-3-0348-8893-6.

Mum63 D. Mumford, Projective invariants of projective structures and applications, Proc. Internat. Congr. Mathematicians (Stockholm, 1962) (Inst. Mittag-Leffler, Djursholm, 1963), 526-530.

Pău08 M. Păun, Regularity properties of the degenerate Monge-Ampère equations on compact Kähler manifolds, Chin. Ann. Math. Ser. B 29 (2008), no. 6, 623-630; http://dx.doi.org/10.1007/ s11401-007-0457-8.

Ser66 J.-P. Serre, Prolongement de faisceaux analytiques cohérents, Ann. Inst. Fourier (Grenoble) 16 (1966), no. 1, 363-374; http://dx.doi.org/10.5802/aif.234.

Sug90 K.-I. Sugiyama, Einstein-Kähler metrics on minimal varieties of general type and an inequality between Chern numbers, Recent Topics in Differential and Analytic Geometry, Adv. Stud. Pure Math., vol. 18, Part I (Academic Press Inc., Tokyo, 1990), 417-443; http: //dx.doi.org/10.1016/B978-0-12-001018-9.50020-8. 


\section{H. Guenancia}

Tak72 F. Takemoto, Stable vector bundles on algebraic surfaces, Nagoya Math. J. 47 (1972), 29-48; http://projecteuclid.org/euclid.nmj/1118798682.

Tsu88 H. Tsuji, Stability of tangent bundles of minimal algebraic varieties, Topology 27 (1988), no. 4, 429-442; http://dx.doi.org/10.1016/0040-9383(88)90022-5.

TY87 G. Tian and S. T. Yau, Existence of Kähler-Einstein metrics on complete Kähler manifolds and their applications to algebraic geometry, Mathematical Aspects of String Theory (San Diego, Calif., 1986), Adv. Ser. Math. Phys., vol. 1 (World Sci. Publishing, Singapore, 1987), 574-628.

UY86 K. Uhlenbeck and S. T. Yau, On the existence of Hermitian-Yang-Mills connections in stable vector bundles, Comm. Pure Appl. Math. 39 (1986), suppl., S257-S293; http://dx.doi.org/ 10.1002/cpa.3160390714.

UY89 _ A note on our previous paper: "On the existence of Hermitian-Yang-Mills connections in stable vector bundles" [Comm. Pure Appl. Math. 39 (1986), S257-S293], Comm. Pure Appl. Math. 42 (1989), no. 5, 703-707; http://dx.doi.org/10.1002/cpa.3160420505.

Yau78 S. T. Yau, On the Ricci curvature of a compact Kähler manifold and the complex MongeAmpère equation. I, Comm. Pure Appl. Math. 31 (1978), no. 3, 339-411; http://dx.doi. org/10.1002/cpa.3160310304.

Henri Guenancia guenancia@math.sunysb.edu

Department of Mathematics, Stony Brook University, Stony Brook, NY 11794-3651 USA 